\title{
Le diable et la critique de la société courtoise dans Gregorius de Hartmann von Aue
}

Der Teufel und die Kritik an der höfischen Gesellschaft in Hartmanns von Aue Gregorius

The devil and criticism of courtly society in Hartmann von Aue's

Gregorius

\section{Patrick Del Duca}

\section{(2) OpenEdition Journals}

Édition électronique

URL : https://journals.openedition.org/ceg/11301

DOI : 10.4000/ceg.11301

ISSN : 2605-8359

\section{Éditeur}

Presses Universitaires de Provence

\section{Édition imprimée}

Date de publication : 1 avril 2012

Pagination : 25-57

ISBN : 0751-4239

ISSN : 0751-4239

\section{Référence électronique}

Patrick Del Duca, « Le diable et la critique de la société courtoise dans Gregorius de Hartmann von Aue », Cahiers d'Études Germaniques [En ligne], 62 | 2012, mis en ligne le 12 octobre 2020, consulté le 07 juin 2021. URL : http://journals.openedition.org/ceg/11301 ; DOI : https://doi.org/10.4000/ceg 11301 


\title{
Le diable et la critique de la société courtoise dans Gregorius de Hartmann von Aue
}

\author{
Patrick DEL DUCA \\ Université Blaise Pascal-Clermont-Ferrand
}

\section{Problématique}

Le Gregorius constitue, avec Le pauvre Henri, l'une des deux légendes composées par Hartmann von Aue à la fin du XII ${ }^{\mathrm{e}}$ siècle. Nous ne savons pas précisément à quelle époque l'auteur allemand a adapté la légende française intitulée La vie du pape Grégoire. La recherche s'accorde généralement à situer la date de composition du Gregorius entre 1190 et 1200, c'est-à-dire durant la même décennie pendant laquelle Hartmann a également transposé en allemand le roman de Chrétien de Troyes Yvain ou le chevalier au lion. Nous ne disposons malheureusement plus du manuscrit qui a servi de source à Hartmann et il existe plusieurs rédactions différentes de la légende française anonyme de saint Grégoire, dont les plus anciennes remontent au XII ${ }^{\mathrm{e}}$ siècle et sont conservées dans six manuscrits. Concernant ces versions anciennes, on peut distinguer deux familles, $\mathrm{A}$ et $\mathrm{B}$, contenues chacune dans trois manuscrits. La version A est globalement plus longue que les textes de B : certains épisodes y sont amplifiés, notamment la description du combat sous les murs de la ville assiégée, l'arrivée de Grégoire à Rome et les retrouvailles finales du pape et de sa mère. Celles-ci ainsi que l'entrée de la mère de Grégoire dans un couvent ne se trouvent que dans A, ainsi que dans $B_{2}$ et $B_{3}$. Il est difficile de savoir comment Hartmann a eu accès à un manuscrit français contenant cette légende ${ }^{1}$. Il est possible que ses mécènes aient été les

1 Pour une analyse des rapports entre les versions françaises et le texte allemand, on pourra se reporter à : Brigitte HERLEM-PREY, Le 'Gregorius' et 'La vie de saint Grégoire'. Détermination de la source de Hartmann von Aue à partir de l'étude comparative intégrale des œuvres. Göppingen, Kümmerle (GAG 215), 1979. 
ducs de Zähringen dont les liens avec la France étaient étroits ${ }^{2}$. Cependant le duché d'Aquitaine, dont Aliénor était l'héritière, peut tout aussi bien trahir l'influence de la famille des ducs de Saxe ${ }^{3}$.

Dans l'étude qu'il a consacrée à cette légende, Ulrich Ernst a clairement mis en évidence le principe sur lequel repose tout le texte : "Diese Struktur ist der Antagonismus von vita carnalis und vita spiritualis, der die dichotomische Konstruktion der Legende des Inzestheiligen konstituiert und die gesamte Dichtung in ihren innersten Bauteilen prägt"4. Cette structure antinomique est soulignée par Hartmann lui-même qui insiste sur

2 Thèse défendue par Volker Mertens, cf. Volker MERTENs, "Das literarische Mäzenatentum der Zähringer", in : Die Zähringer. Eine Tradition und ihre Erforschung, éd. par Karl Schmid, Sigmaringen, Thorbecke Verlag, 1986, p. 117-134; Volker MERTENS, Gregorius Eremita. Eine Lebensform des Adels bei Hartmann von Aue in ihrer Problematik und ihrer Wandlung in der Rezeption. München, Artemis, 1978, p. 25-26.

Plusieurs indices plaident effectivement en faveur de cette hypothèse. Les ducs de Zähringen étaient également recteurs de Bourgogne et disposaient sans aucun doute des moyens financiers nécessaires à l'acquisition des manuscrits contenant les textes que Hartmann a adaptés en allemand (Erec et Enide et Yvain ou le chevalier au lion de Chrétien de Troyes, ainsi que cette légende). La famille ducale pouvait constituer un véritable pont culturel entre l'Allemagne et la France : le duc Berthold IV de Zähringen (né vers 1125 et mort en 1186) épousa en secondes noces, en 1183, Ide de Boulogne, fille de Mathieu d'Alsace et Marie de Blois. L'oncle d'Ide de Boulogne était le comte Philippe de Champagne pour lequel Chrétien de Troyes composa son Perceval entre 1181 et 1191. De plus, la mère de Berthold IV, Clémence de Luxembourg-Namur, parlait français. Nous aurions donc là un itinéraire propice à un transfert culturel de Francie en Germanie. L'allusion que fait Hartmann à la mort de son suzerain dans l'une de ses chansons de croisade ("Maintenant que la mort m'a privé de mon seigneur, plus rien ne m'importe de ce qui se passe dans le monde") est également compatible avec l'année de décès de Berthold IV. Le lien avec la France ne se rompt pas avec la mort de Berthold IV : Berthold V (1160-1218), le fils qu'il avait eu avec sa première femme Heilwich de Frohbourg, épousa vers 1200 Clémence d'Auxonne, fille d'Etienne III de Bourgogne, comte d'Auxonne. Les ducs de Zähringen ont également encouragé les arts : selon l'écrivain Rudolf von Ems, Berthold von Herbolzheim a composé un roman d'Alexandre pour le "noble Zähringer", sans doute Berthold V ou son père. Enfin, les armoiries attribuées à Hartmann dans le Grand Chansonnier de Heidelberg sont composées d'alérions blancs sur fond d'azur et pourraient avoir été formées à partir du blason des ducs de Zähringen, représentant une aigle de gueules, déployée sur fond d'or.

3 Aliénor d'Aquitaine, première femme du roi français Louis VII, se maria en secondes noces avec le futur roi d'Angleterre : l'union eut lieu en 1152, deux ans plus tard son époux coiffait la couronne d'Angleterre sous le nom d'Henri II. En 1168, le duc de Saxe Henri le Lion épousa Mathilde, fille d'Henri II Plantagenêt et d'Aliénor. Après avoir été banni de l'empire par Frédéric Ier Barberousse, Henri le lion passa lui-même trois années d'exil en Angleterre (de 1182 à 1185), chez son beau-père Henri II. On notera également que le plus jeune fils d'Henri le Lion, Guillaume de Lunebourg, né en Angleterre en 1184, a fait traduire le Gregorius de Hartmann en latin par l'évêque Arnold de Lubeck. Il n'est pas impossible que la légende française ait été une commande d'Aliénor d'Aquitaine elle-même : accusée par ses contemporains d'avoir eu des relations incestueuses avec son oncle Raimond d'Antioche puis avec Geoffroy d'Anjou, père de son deuxième mari Henri II, elle aurait peut-être voulu faire œuvre de pénitence en commandant cette légende.

4 Ulrich ERNST, Der 'Gregorius' Hartmanns von Aue. Theologische Grundlagen legendarische Strukturen - Überlieferung im geistlichen Schrifttum (Ordo, Bd. 7, Studien zur Literatur und Gesellschaft des Mittelalters und der frühen Neuzeit), Köln/Weimar/Wien, Böhlau, 2002, p. 3. 
l'incompatibilité fondamentale existant entre l'âme et le corps (Gregorius, v. 2655-2662). U. Ernst démontre que cette légende développe de manière très cohérente l'opposition entre la gloria mundi et la gloria Dei $i^{5}$, entre les valeurs profanes propres au monde courtois et les valeurs chrétiennes qui se manifestent à travers la fuga mundi, la vie contemplative ou l'érémitisme. Nous tenterons d'analyser dans cet article quelle fonction remplit le diable dans la légende allemande, et comment le rôle du Malin s'intègre dans la structure d'ensemble du texte et participe de son sens global.

Poser la question du diable revient à s'interroger sur le mal, la source du mal et, l'un des problèmes fondamentaux posés par Hartmann, sur les raisons qui expliquent pourquoi Dieu tolère l'action du diable. Pour la patristique, l'action du diable permet de mettre les fidèles à l'épreuve. Ainsi, selon saint Grégoire, si Dieu déclare au diable au sujet de Job : "Voici, tout ce qui lui appartient est en ton pouvoir", c'est que par une puissance mystérieuse, il souhaite "donner libre cours aux assauts du malin contre les fidèles, pour les éprouver" ". Cependant, la puissance du diable demeure limitée et sous le contrôle de Dieu : "Pour Dieu, dire : Seulement, ne porte pas la main sur lui, c'est aussi, dans la permission qu'il donne, contenir la tentation en en modérant la violence" " . Dieu concède donc au Malin la permission d'éprouver la vertu des croyants ${ }^{8}$ en même temps qu'il lui interdit de porter la main sur eux. Le pouvoir du diable et les pièges qu'il tend aux hommes s'inscrivent dans le plan divin, dans la Providence. Le diable n'aura donc de cesse de parcourir le monde en quete de victimes, cachant toujours ses ruses et sa malice aux yeux des hommes ${ }^{9}$, enviant leurs progrès, sondant les failles qui s'ouvrent à la réprobation, brûlant de faire le mal pour affliger les bons ${ }^{10}$.

Le problème de la faute tel qu'il a très souvent été posé par la critique à propos de Gregorius mène, comme le souligne Ulrich Ernst, dans une impasse $^{11}$. La légende de Hartmann s'inscrit dans une perspective beaucoup

5 Ibid., p. 5-17.

6 GrÉGOIRE Le Grand, Moralia in Job, PL vol. 75, col. 561C : "Dicere Dei est : Ecce universa quce habet, in manu tua sunt, ad probationem fidelium, contra eos occulta vi incursum illum suce malitice relaxare".

Traduction d'après : Morales sur Job, introduction et notes de Dom Robert GILLET, traduction de Dom André de Gaudemaris, Paris, Editions du Cerf (Sources Chrétiennes 32), 1950, Livre 2, VII, 12.

7 GrÉGOIRE Le Grand, Moralia in Job, PL vol. 75, col. 561C : "Dicere Dei est : Tantum in eum ne extendas manum tuam, ab immoderatce tentationis impetu eum etiam permittendo restrigenre".

8 Ibid., PL vol. 75, col. 561B : "Tentandam eorum innocentiam ei concedendo permittit".

9 C'est pourquoi le secret tient une telle place dans la légende de Gregorius : le secret de l'inceste, celui qui entoure la naissance de l'enfant, les pêcheurs qui tentent de cacher l'enfant à l'abbé, l'abbé qui achète le silence des pêcheurs et qui ne dévoile pas à Gregorius ses origines véritables, Gregorius qui, de retour en Aquitaine, dissimule les tablettes qui révèlent son identité et qui auraient permis d'éviter la deuxième catastrophe...il est évident que la culture du secret dans la légende s'inscrit dans le plan du diable et participe de la chute du héros. Cf. Ulrich ERNST, Der 'Gregorius' Hartmanns von Aue. Theologische Grundlagen, p. 18 ss.

10 Cf. GRÉGOIRE LE GRAND, Moralia in Job, PL vol. 75, col. 562A.

11 Ulrich ERNST, Der 'Gregorius' Hartmanns von Aue. Theologische Grundlagen, p. 1. 
plus large que le simple problème de la responsabilité personnelle. Elle est révélatrice d'une perspective chrétienne et rigoriste, d'une idéologie qui va à l'encontre les valeurs profanes et mondaines. Pour pouvoir en appréhender les différentes facettes et en dégager l'originalité, il nous faudra non seulement comparer régulièrement le texte allemand aux versions françaises dont nous disposons aujourd'hui, mais nous devrons également replacer l'œuvre dans le contexte socio-historique qui lui est propre. La fonction du diable dans Gregorius et le traitement qui lui est réservé, en comparaison de l'image qui est donnée du diable dans La vie du pape Grégoire, sont en effet révélateurs d'une nouvelle orientation de la légende.

\section{Dieu et le diable dans les versions françaises de la légende et dans le Gregorius de Hartmann von Aue}

La légende française relate la vie de Grégoire, né des amours incestueuses du jeune comte d'Aquitaine et de sa sœur:

Un puissant comte d'Aquitaine, à l'article de la mort, convoque ses barons et, devant eux, demande à son fils de veiller sur sa sœur. Avant d'expirer, le comte, regrette amèrement de ne pas avoir marié sa fille : "Se je l'eüsse mariee / De rien ne fust m'arme grevee"12 ( $\mathrm{A}_{1}$, v. 89-90, "Si je l'avais mariée, mon âme ne serait nullement attristée"). Il déplore que celle-ci demeure toute "desconseillee" ( $\mathrm{A}_{1}$, v. 93) et toute "esgaree" ( $A_{1}$, v. 105), qu'elle soit déconcertée, dépourvue d'aide et de conseils. Le frère s'occupe loyalement de sa sœur, ne la quittant jamais, et la tient en grand honneur. Cette relation fraternelle déplaît profondément au diable qui, profitant de l'amour et de l'intimité des deux êtres, fait en sorte que le frère s'éprenne de sa sœur et la viole. L'ennemi s'en réjouit et croit déjà les avoir condamnés à l'enfer $\left(A_{1}, v\right.$. 203-208). De cette relation incestueuse naît bientôt un fils, dont Dieu fera un saint homme et un pape. Le diable ignore cependant tout de ce "saintisme engendrement" $\left(\mathrm{A}_{1}, \mathrm{v}, 216\right)$. Il s'affaire à aiguillonner ces deux victimes afin qu'elles continuent à vivre dans le péché, "mais il fut finalement bien trompé" ( $A_{1}, v .222$, "Mais puis i fu mal enginés"). Dès que la dame se sait enceinte, elle déclare qu'elle ne supprimera pas le fruit que Dieu a mis en elle. Les deux enfants demandent le conseil d'un vieux baron, fidèle vassal et ami de leur père défunt. Le baron recueille la demoiselle afin qu'elle puisse accoucher chez lui en secret et ordonne au jeune homme de partir en pèlerinage à Jérusalem afin de sauver son âme. La jeune femme donne naissance à un très beau garçon et exige du vieux baron et de sa femme qu'ils fassent ce qu'elle demandera, sans quoi elle refusera de s'alimenter. Ceux-ci, après avoir cru que la mère voulait tuer l'enfant, finissent par se conformer à ses désirs et trouvent un berceau pour le nourrisson. La jeune mère y dépose l'enfant, elle rajoute près de sa tête quatre marcs d'or, du sel pour indiquer qu'il n'est pas baptisé $^{13}$, ainsi qu'un riche drap d'or et de soie. Sous les pieds du nourrisson,

12 La légende française est citée d'après : Hendrik Bastian Sol (éd.) : La vie du pape saint Grégoire. Huit versions françaises médiévales du bon pécheur. Amsterdam, Rodopi, 1977.

13 Cette tradition remonte à Saint Augustin : l'imposition du sel est un signe destiné aux catéchumènes. Il s'agit d'un exorcisme visant à chasser le Malin en attendant que les catéchumènes soient baptisés : "Ce qu'ils reçoivent, bien que ce ne soit pas le corps du Christ, 
elle place dix marcs d'argent, destiné à son éducation, et des tablettes d'ivoire sur lesquelles elle écrit qu'il est né "par peché et par folie" $\left(A_{1}\right.$, v. 514) d'une relation entre un frère et une sœur et qu'il est de "haut parage" $\left(A_{1}, v .519\right)$. Le vieux couple de nobles place le berceau dans un tonneau et va le déposer dans un bateau vide qu'ils abandonnent aux flots, tandis que la mère, désespérée, croit mourir de douleur. Bientôt, elle apprend la mort de son frère qui, dès qu'il l'eut quittée, fut pris d'un mal qui l'emporta en une journée. A la demande des barons, la demoiselle ensevelit son frère et prend sa place à la tête de l'héritage laissé par leur père. Convoitée par de nombreux comtes, elle refuse de se marier à aucun d'entre eux jusqu'au jour où un puissant duc, venu d'Italie et également éconduit par la comtesse, lui fait la guerre et ravage son pays.

L'enfant, quant à lui, vogue au gré des flots, comme le veut la "fortune" $\left(\mathrm{A}_{1}\right.$, v. 772), frôlant de grands périls et la mort. Il est conduit par Dieu qui le protège et lui permet d'arriver à proximité d'un port où deux pêcheurs, appartenant à une abbaye, n'ont pas réussi à jeter leurs filets à cause de la tempête. Les deux pêcheurs, deux frères dont l'un est pauvre et l'autre riche, recueillent le berceau et le ramènent sur la rive où les attend l'abbé. Celui-ci découvre l'enfant que les pêcheurs tentent de dissimuler et le confie au pêcheur pauvre afin qu'il l'élève. Il lui donne les dix marcs d'argent afin que le pêcheur se taise et fasse passer le nourrisson pour son petit-fils. L'abbé conserve les tablettes, l'or ainsi que l'étoffe précieuse et baptise l'enfant dès le lendemain, devenant son parrain. A cinq ans, Grégoire entre au couvent. Le pêcheur, pressé de questions par sa femme qui veut savoir d'où vient cet argent, ne tient pas parole et lui révèle comment Grégoire a été trouvé. Alors qu'il a quinze ans et qu'il joue avec les enfants du pêcheur, Grégoire saisit l'un de ses demi-frères par les cheveux et l'abat par terre $\left(A_{1}, v .1004\right)$. Le garçon blessé va se plaindre auprès de sa mère qui, sous l'emprise de la colère, injurie Grégoire, le traitant de misérable et d'enfant trouvé. Grégoire demande alors à l'abbé l'autorisation de quitter ce pays, car il ne supporte pas la honte dont il est l'objet. L'abbé tente de détourner Grégoire de son projet et lui assure qu'à sa mort c'est lui que les moines éliront à la tête du monastère. Grégoire refuse cette offre et annonce son désir de devenir chevalier, car tout son cœur et toute sa vie sont tournés vers la chevalerie. Là encore, l'abbé tente de le dissuader de rejoindre le corps des chevaliers "que molt mauvaise est lor vie" $\left(A_{1}, v .1128\right)$, arguant qu'il perdra son âme $\left(A_{1}, v\right.$. 1208). Il cède malgré tout et procure à son filleul un équipement de chevalier. Une dernière fois, il tente de le retenir en lui promettant une terre et un mariage avec une femme de haute naissance. Alors que Grégoire déclare qu'il ne sait de quel lignage il est issu, l'abbé va chercher les tablettes d'ivoire. Bouleversé par la révélation du secret de sa naissance, Grégoire refuse de rester. L'abbé lui a fait don d'un bliaut taillé dans l'étoffe précieuse que contenait son berceau.

Grégoire s'embarque dans un bateau afin de traverser la mer. Le diable, qui veut le damner, le ramène au pays de sa mère, la comtesse d'Aquitaine. Après avoir été présenté à la comtesse, Grégoire entre à son service et la délivre des assauts du duc romain qui avait mis le siège à sa capitale. Le duc, fait prisonnier par Grégoire, doit s'engager à laisser la comtesse définitivement en paix. Celleci a bien remarqué le bliaut que porte Grégoire mais pense qu'il ne s'agit que d'une coïncidence. Sur les conseils de ses barons, elle épouse le chevalier qui les a délivrés du duc. Ceci entraîne la grande joie du diable qui, après avoir uni le frère à la sœur, est parvenu à unir le fils à la mère. Chaque jour, Grégoire se rend

est néanmoins saint, $[\ldots]$ parce que c'est un sacrement" (De peccatorum meritis et remissione, II, 26, 42 ; "et quod accipiunt, quamvis non sit corpus Christi, sanctum est tamen, [...] quoniam sacramentum est", PL vol. 44, col. 176). Ce signe pourra être renouvelé plusieurs fois avant le baptême effectif. 
dans sa chambre où il consulte en secret ses tablettes d'ivoire. Il en ressort toujours tellement abattu que sa tristesse éveille la curiosité d'une femme de chambre qui avertit la comtesse. Celle-ci découvre les tablettes et, profondément bouleversée par cette nouvelle faute, révèle la vérité à son fils. Le fils, qui a jadis fréquenté l'école du monastère, instruit sa mère sur la façon d'obtenir le pardon de Dieu et revêt lui-même la haire du pénitent. Il renonce au monde, à la richesse, et quitte le pays, n'emmenant avec lui que ses tablettes.

Au bout de trois jours, il parvient dans une région isolée au bord de la mer. Il n'y a là qu'une cabane de pêcheur habitée par un couple pauvre. Le pêcheur, un méchant homme qui prend Grégoire pour un brigand, lui refuse le gîte, puis, après l'intervention de sa femme, lui accorde l'hospitalité pour la nuit. Le lendemain, ayant appris que Grégoire recherche un ermitage, il l'emmène sur une île déserte et l'enchaîne à un rocher avant de jeter la clé des chaînes à la mer. Ne survivant que grâce à l'eau qui tombe du ciel et à l'aide du Christ, Grégoire passe dix-sept ans dans la solitude la plus totale et un dénuement extrême. Ses vêtements ont pourri de telle sorte que seuls ses poils le protègent du froid ou de la chaleur. A Rome, le pape vient de mourir et les cardinaux apprennent par un ange que son successeur doit être un pénitent qui répond au nom de Grégoire. Les messagers partis à la recherche de cet inconnu passent une nuit chez le pêcheur qui a abandonné Grégoire sur l'île. Il leur offre comme repas un grand poisson et découvre, en l'ouvrant, la clé qu'il avait jetée à la mer dix-sept ans plus tôt. Pris de repentir, il relate aux envoyés de Rome ce qui s'est passé jadis. Dès le lendemain, il les conduit au rocher où est toujours attaché Grégoire, qui a maintenant pris l'apparence d'une bête. Celui-ci, après avoir appris comment la clé a été retrouvée, consent à devenir pape et se rend à Rome (fin de $\mathrm{B}_{1}$ ). La Comtesse d'Aquitaine se rend également à Rome pour obtenir le pardon du pape. Après la confession, Grégoire se fait reconnaître de sa mère. Il la fait entrer dans un couvent afin qu'elle expie ses péchés et que son âme soit sauvée (fin de $A_{1}, A_{2}, A_{3}, B_{2}, B_{3}$ ).

Dans un article paru en $1988^{14}$, Anita Guerreau-Jalabert a démontré que la légende du pape Grégoire est en premier lieu une dénonciation de la politique matrimoniale féodale telle qu'elle était pratiquée dans le duché d'Aquitaine au XII ${ }^{\mathrm{e}}$ siècle. En effet, la première erreur commise par le père des deux enfants incestueux réside dans le "refus réitéré du mariage exogamique 'normal', ce qui les précipite dans une pratique de l'inceste que l'auteur du récit n'a pas choisi de présenter en demi-teintes" ${ }^{\prime 15}$. Il a failli à ses devoirs de père et de suzerain en ne se montrant pas assez prévoyant et "a gravement mis en cause la reproduction normale de son 'lignage' - et de son pouvoir -, mais également, dans le même temps, celle de tout le groupe des barons" ${ }^{\text {"16. }}$. Le comte a donc, par cette négligence fatale, bloqué la reproduction exogamique de son lignage et mis en péril les liens de vassalité qui assurent la bonne cohésion et la paix de la société féodale. De la même façon, la comtesse va, en guise de pénitence, refuser toute nouvelle union exogamique. Comme l'a noté A. Guerreau-Jalabert, ce comportement est là encore en totale décalage

14 Anita GUERREAU-JALABERT, "Inceste et sainteté. La vie de saint Grégoire en français (XII ${ }^{\mathrm{e}}$ siècle)", in : Annales. Economies, Sociétés, Civilisation, $43^{\mathrm{e}}$ année, $\mathrm{n}^{\circ}$ 6, 1988, p. 12911319.

15 Ibid., p. 1299.

16 Ibid., p. 1299. 
avec les normes de la société laïque du Moyen Âge : en choisissant la chasteté elle se comporte comme une veuve, assimilant "par son attitude relations incestueuses et mariage légitime" $"$. Elle opère donc un choix inadéquat dont les conséquences sont la dévastation de ses terres par le duc romain qui souhaitait l'épouser. La culture du secret, liée à l'inceste, qui entoure la conception de Grégoire ainsi que sa naissance vont, selon l'auteur de l'article, à l'encontre des règles de l'Eglise qui prônent la publicité de tels rites afin de garantir la légitimité du lignage. Par ailleurs, aucune place n'est accordée à l'Eglise lors de la conclusion du mariage entre le jeune chevalier et sa mère : seuls les barons, inspirés par le diable, cautionnent cette union. Ce texte se fait donc l'écho de la condamnation d'une "conception beaucoup trop exclusivement laïque du contrôle des relations de parenté (filiation et plus encore alliance) dans l'aristocratie" ${ }^{\prime 1}$. Un tel repli de la société aristocratique sur elle-même à travers la pratique du mariage endogamique n'aura que des conséquences néfastes, entraînant une rupture de la reproduction normale du lignage et empêchant un bon fonctionnement de la société féodale (l'absence d'un seigneur entraînant la guerre). A cette conception erronée d'une pratique matrimoniale oublieuse des principes de l'Eglise la légende oppose, selon Anita Guerreau-Jalabert, la parenté spirituelle entre Grégoire et l'abbé. En effet, c'est bien Dieu qui conduit le jeune Grégoire au monastère où il sera baptisé par l'abbé, son parrain qui lui invente une nouvelle famille, celle du pêcheur. Par le baptême, Grégoire reçoit un nom, est intégré "dans le réseau de la parenté spirituelle chrétienne", ce qui "souligne le caractère absolument positif de toute filiation artificielle [...] et sa prédominance 'logique' sur la filiation réelle"19. A la parenté charnelle imposée, d'origine diabolique, répond donc une parenté spirituelle choisie. Le stade ultime de cette parenté spirituelle ne sera cependant atteint que sur l'île déserte, dans une union mystique avec Dieu : après avoir, à travers l'inceste avec sa mère, "choisi la parenté réelle au détriment de la parenté spirituelle" et après s'être "lui-même remis au pouvoir du diable", Grégoire atteint une relation spirituelle qui dépasse toutes les autres, l'union avec Dieu. Cet épisode fait donc la "démonstration de la supériorité absolue de la parenté spirituelle - de nature divine - sur la parenté réelle - liée aux agissements du diable, 20 .

En transposant ce récit à l'Allemagne de la fin du XII ${ }^{\mathrm{e}}$ siècle, Hartmann von Aue a changé de nombreuses données du récit français. Même si le cadre socio-historique n'est pas fondamentalement différent de celui qui caractérisait la légende française - nous retrouvons dans l'adaptation allemande l'opposition marquée entre une conception laïque et féodale du mariage et l'idéalisation de la parenté spirituelle et de la relation avec Dieu -

17 Ibid., p. 1300.

18 Ibid., p. 1301.

19 Ibid., p. 1303.

20 Ibid., p. 1304. 
de nombreux détails ont été changés qui infléchissent le sens du texte et la fonction remplie par le diable.

Dans le texte français, l'irruption du diable ne paraît guère surprenante : qu'y a-t-il d'anormal à ce que le diable intervienne dans les affaires humaines alors que dans cette société pervertie les valeurs élémentaires ne sont plus respectées ? Quand un père néglige ses devoirs élémentaires, omettant d'organiser le mariage de ses enfants, laissant sa fille sans autre protection que celle de son frère, l'intervention de l'ennemi de l'humanité est finalement logique et prévisible. Qu'en est-il dans le Gregorius ${ }^{21}$ ? Il semble tout d'abord que l'auteur allemand ait à cœur de nous présenter une société beaucoup plus "normale", dans laquelle les rapports entre les individus ne sont pas aussi pervertis, une société avec laquelle le public courtois de Hartmann aura plus de facilités à s'identifier ${ }^{22}$. Ainsi l'auteur réduit-il tout d'abord la responsabilité du père. En effet, lorsqu'il meurt, ses enfants n'on que dix ans (v. 188), ils ne sont donc pas nubiles. On peut dès lors reprocher au père d'avoir omis de préparer leurs mariages, de n'avoir pas songé à de nouvelles alliances avec d'autres familles, mais on ne peut plus lui faire grief d'avoir refusé de les marier. Les paroles que tient le duc allemand sont d'ailleurs beaucoup moins empreintes de repentir et de tristesse que celles du comte français :

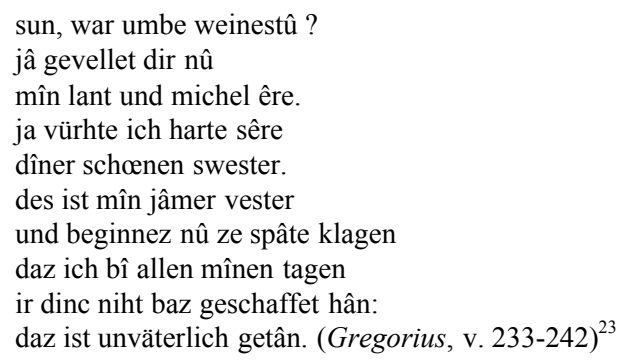

La faute commise par le père est donc moindre que dans la source. En évoquant la faute commise par le père, l'adaptation allemande ne met nullement l'accent sur le désarroi de la jeune fille, ni sur le fait qu'elle soit dépourvue d'aide et de conseil, mais au contraire - nous y reviendrons en détail - sur les honneurs qui reviennent de droit au fils. Comme dans le texte français, on insiste sur le dévouement dont le frère fait preuve envers sa sœur

21 Nous citerons le texte allemand de la légende d'après l'édition suivante : Hartmann von Aue, Gregorius, Der arme Heinrich, Iwein, herausgegeben und übersetzt von Volker MERTENS (Bibliothek der Mittelalters 6), Frankfurt am Main, Deutscher Klassiker Verlag, 2004.

22 Ceci permet à Hartmann de faire de sa légende un exemple ayant une valeur générale. Ainsi l'auteur ajoute-t-il une mise en garde destinée à chaque homme afin qu'il n'ait pas de relation trop intimes avec une sœur ou une nièce (Gregorius, v. 415-420)

23 "Mon fils, pourquoi pleures-tu puisque maintenant mon pays et de grands honneurs vont te revenir? En fait, c'est pour ta sœur, si belle, que je me fais vraiment du souci. Je regrette profondément, mais mes regrets arrivent trop tard, de ne pas avoir mieux pourvu à son avenir de mon vivant. Je n'ai pas agi en père". 
et sur le bonheur qu'ils éprouvent à être ensemble. C'est justement ce grand bonheur qui déplaît au diable de telle sorte qu'il transforme l'amour loyal du frère en de mauvaises pensées :

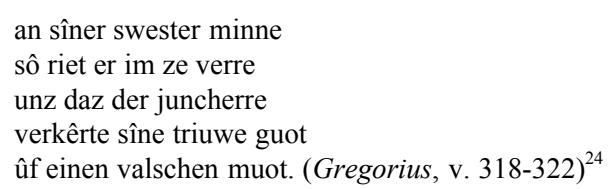

Là encore, la responsabilité des individus est très largement atténuée. Si l'influence néfaste du diable est déjà bien présente dans le récit français, elle paraît encore plus déterminante dans l'adaptation : la volonté du jeune homme et son libre arbitre semblent ici totalement annihilés si bien qu'il devient l'objet du diable. Celui-ci profite de l'innocence des deux enfants pour les manipuler à sa guise. Il abuse le jeune garçon qui agit "sur le conseil du diable" (v. 339, "durch des tiuvels rât") et endort la méfiance de la jeune fille :

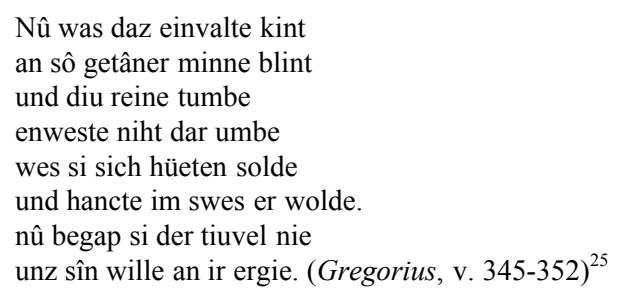

Dans la légende française, le Malin profite des baisers que tous deux se donnent trop souvent pour que le frère s'éprenne de la sœur $\left(\mathrm{A}_{1}\right.$, v. 157-160). Dans l'adaptation de Hartmann, c'est le diable qui fait en sorte que le jeune homme enlace sa sœur en dormant et que celle-ci colle sa bouche et ses joues à son frère :

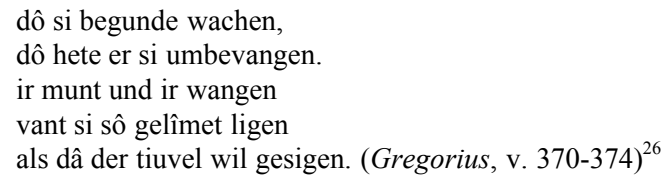

De la même façon, lorsque la jeune femme prend plaisir à s'adonner régulièrement à ce péché, c'est encore "l'excitation provoquée par le diable"

24 "Il lui inspira si longtemps du désir pour sa sœur que la loyale amitié du jeune seigneur fit place à de perfides intentions".

25 "Alors, la jeune enfant était aveugle face à un amour d'une telle nature et, pure et naïve, elle ne savait pas de quoi elle devait se garder si bien qu'elle le [= son frère] laissa faire ce qu'il voulut. A ce moment, le diable ne lâcha pas prise jusqu'à ce qu'il l'ait soumise à sa volonté".

26 "Lorsqu'elle se réveilla, il la tenait enlacée. Elle vit que sa bouche et ses joues étaient collées à lui, comme c'est le cas lorsque le diable veut triompher". 
(v. 401, “der tiuvelschünde luoder") qui est à l'œuvre. L'œuvre allemande ne fait nulle mention d'une relation qui serait en elle-même pervertie, elle souligne au contraire la loyauté et la bonté du frère ainsi que l'irruption du diable qui lui inspire une passion violente. Le narrateur énumère les quatre facteurs qui sont à l'origine de cette passion : il évoque tout d'abord l'amour qui tourmente les sens du jeune homme, en second lieu la beauté de sa sœur, en troisième lieu la fausseté du diable et enfin la jeunesse du frère (v. 327, "sîn kindheit"). Ces différents facteurs, loin d'être indépendants les uns des autres, constituent un tout : l'amour, la beauté de la jeune femme et la jeunesse, c'est-à-dire la naïveté, du garçon, sont en réalité les adjuvants du Malin "qui se sont alliés contre le jeune homme et avec le diable" (v. 328, "diu ûf in mit dem tiuvel streit"). Parallèlement, la figure de la sœur n'est pas aussi ambigüe que dans l'œuvre française : elle n'exerce aucun chantage envers les deux nobles qui l'ont accueillie et ne menace pas de se laisser mourir de faim. C'est d'ailleurs ensemble que tous trois vont confier le nourrisson aux flots (v. 767-784). Faut-il en conclure que l'action du diable sert à disculper les hommes? Ce serait sans doute aller trop vite en besogne, même s'il est vrai que Hartmann prend soin de ne pas présenter ses personnages sous un trop mauvais jour. Cette tendance à la revalorisation des personnages se retrouve dans la plupart de ses œuvres et n'est pas propre au Gregorius.

La puissance du diable semble être beaucoup plus importante dans l'œuvre allemande que dans le récit français. Daniel Rocher a souligné à juste titre que le pessimisme de Hartmann se démarque radicalement de l'optimisme dont est empreinte la légende française ${ }^{27}$. En effet, dans le texte français, l'inceste commis par les deux enfants s'inscrit, sans que personne ne le sache, dans le plan divin : de cette union naîtra le très saint Grégoire ${ }^{28}$. D. Rocher démontre que l'on retrouve ici le thème de la felix culpa propre à la liturgie pascale ${ }^{29}:$ ce thème fait partie de l'Exultet chanté durant la veillée du samedi saint: "O felix culpa quae talem et tantum meruit habere redemptorem" ("Heureuse faute d'Adam qui nous a valu un rédempteur"). Même le péché originel participe du bien et de la rédemption finale de l'humanité. Comme toute œuvre de Satan, il s'inscrit dans le plan de Dieu. Cette vision optimiste de l'auteur de la Vie du pape Grégoire, très proche de la conception de la plupart des pères de l'Eglise, constitue une sorte de Théodicée, une justification du mal sur terre, et est confirmée par le fait que

27 Daniel Rocher, "Das Motiv der 'felix culpa' und des betrogenen Teufels in der 'Vie du pape Grégoire' und in Hartmanns 'Gregorius'”, in : Germanisch-romanische Monatsschrift, 1988 , vol. 38 , p. $57-66$

28 Ibid., p. 58 : "Im Moment, wo Gregorius' Eltern in den Augen der Menschen - wenn sie davon wüßten - eines der schlimmsten sexuellen Vergehen, die man sich denken kann, begehen, tun sie, ohne es zu wissen, etwas Heiliges, ja Heiligstes (saintisme ist ein Superlativ), indem sie einen 'Heiligen' zeugen, der alles wiedergutmachen wird'.

29 Ibid., p. 58 : "Und hier dürfen wir wohl einen Anklang an den berühmten Ausruf der christlichen Liturgie vernehmen, die felix culpa der Osterhymne Exultet, die in der Osternacht gesungen wird". 
la puissance du diable est limitée. Le diable est dupé et ne se doute pas que de l'union du frère et de la sœur naitra un saint. Sa victoire est donc de courte durée et est d'emblée vouée à se transformer en un échec cuisant. De la même façon, lorsque Grégoire apprend qu'il a épousé sa mère, il ne désespère pas mais s'adresse au diable pour le défier parce qu'il sait que Dieu est à ses côtés. L'inceste devient la condition nécessaire au rachat, à la rédemption et, finalement, à l'union avec Dieu. Le diable n'est donc que l'instrument de Dieu et son rayon d'action demeure limité. Daniel Rocher a également noté que ces deux passages, qui annoncent la défaite du diable, ont été supprimés par Hartmann : rien dans le texte allemand n'indique que le diable, le trompeur par excellence, est lui-même mystifié. Le défi lancé au Malin est lui aussi supprimé et remplacé par la colère qu'éprouve Gregorius envers Dieu $^{30}$ : "sînen zorn huop er hin ze gote" (v. 2068 : "Il donna libre cours à sa colère envers Dieu").

Il semble, d'après D. Rocher, qu'il y ait dans le texte allemand une contradiction entre l'aide et la protection que Dieu apporte régulièrement à Gregorius et l'influence du diable. Faut-il en conclure, comme le fait l'auteur de l'article, qu'il serait erroné de vouloir faire rentrer l'œuvre de Hartmann dans un carcan de logique et de cohérence ${ }^{31}$ ? Il y aurait un aspect ludique qu'il ne faudrait pas négliger, et l'alternance des passages relatifs à l'aide de Dieu ou à l'influence du diable ne ferait finalement que renvoyer à la même idée que celle développée dans l'œuvre française : quoi qu'il fasse, l'action du diable vient s'inscrire dans le plan divin. L'action du diable ne sert qu'à mettre l'homme à l'épreuve, une épreuve terrible et cruelle dont le "bon pécheur" sort finalement grandi. Ceci correspond effectivement à la conception que le christianisme a de l'action du diable et dont la figure biblique de Job constitue un parfait exemple.

En y regardant de plus près, il n'est cependant pas certain qu'il y ait une contradiction entre l'action bénéfique de Dieu et les ruses du diable. En effet, juste avant que le frère ne couche avec sa sœur, le narrateur pose l'une des questions essentielles que soulève le texte allemand :

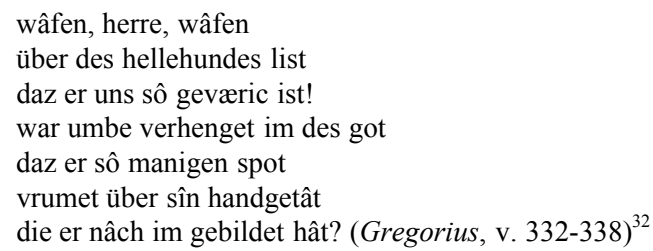

30 Ibid., p. 63 : "Dazu hat Hartmann die Gebärde, mit der Grégoires Dank an Gott anhub Envers le ciel tendi ses mains ('er hob seine Hände gen Himmel'), ins Abstrakte und in ihr Gegenteil verwandelt".

31 Ibid., p. 64 : "Man darf Hartmann nicht in eine strenge Logik einzwängen wollen".

32 “Quel malheur, seigneur Dieu, quel malheur que les subterfuges du monstre de l'enfer soit si dangereux pour nous. Pourquoi Dieu a-t-il permis que le diable puisse se jouer ainsi de ceux que le Seigneur a créés de sa main et qu'il a faits à son image?" 
La question fondamentale posée par le texte de Hartmann est celle de la justification et du sens du mal sur terre. Pourquoi Dieu tolère-t-il le mal ?

A cette question, le narrateur, à l'instar des Pères de l'Eglise, ne peut donner de réponse. De la même façon, saint Augustin affirme dans La Cité de Dieu $(\mathrm{XX}, 2)^{33}$ : "Ainsi dans les mystères mêmes de sa justice, Dieu cache un enseignement salutaire. Nous ne savons pas en effet par quel jugement de Dieu, ce juste est pauvre, et ce méchant est riche ; celui-ci, dans la joie, qui, selon nous mériterait d'expier, par de cruelles douleurs, la corruption de ses mœurs; celui-là dans la tristesse, à qui une vie exemplaire devrait assigner le bonheur pour récompense". Il semble que ce soit là cette cécité inhérente à la conditio humana, cette incapacité à percer les desseins de Dieu et surtout à distinguer le bien du mal, que Hartmann veuille mettre en évidence. L'homme, prisonnier de sa condition, ne peut finalement différencier la volonté de Dieu des ruses du diable. A chaque fois qu'il croit agir en adéquation avec la volonté divine et les préceptes de l'Eglise, il se trompe et fait, sans le savoir, l'œuvre du diable. Ainsi, alors que le père, mourant, s'apprête à prendre définitivement congé de ses enfants, il adresse à son fils une série de conseils, véritable miroir des princes, afin que le jeune homme devienne un bon seigneur et fasse preuve de loyauté, de constance, de générosité, d'humilité, de force envers les puissants et de bonté envers les humbles (v. 244-265). Il lui demande également de s'occuper fraternellement de sa sœur, ce que le jeune homme tentera effectivement de faire, prenant soin d'elle "du mieux qu'il pouvait et aussi loyalement que possible" (v. 277 s., "und pflac ir sô beste mohte / als sînen triuwen tohte"). Cette bonne volonté et ces efforts sincères furent cependant tous vains, si bien que le narrateur affirme, non sans ironie, que le jeune homme fit décidément "preuve de trop de sollicitude" (v. 396, "dâ was triuwen alze vil").

Cette inadéquation entre la bonne volonté affichée des hommes et le résultat finalement obtenu est particulièrement visible lors de l'épisode qui retrace la relation incestueuse que Gregorius a avec sa mère. Lorsqu'il quitte l'île et le monastère où l'abbé a tenté en vain de le retenir, il s'en remet à Dieu, espérant que Celui-ci le guidera afin qu'il puisse apprendre d'où il vient et qui il est : il lève les bras vers le ciel et lance une prière à Dieu afin que son

Cet aspect sera développé une seconde fois par la suite : après que la mère de Gregorius a découvert qu'elle a épousé son fils, le narrateur ajoute que "Dieu a à nouveau permis au diable d'user d'artifices si bien qu'elle était tombée une seconde fois dans le gouffre des péchés" (v. 2495-2498, "sît er des tiuvels râte / nû aber verhenget hâte / daz si an der sünden grunt / was gevallen anderstunt").

33 Cité d'après : Saint Augustin, La Cité de Dieu, traduction du latin de Louis Moreau (1846) revue par Jean-Claude ESLIN, introduction, présentation et notes par Jean-Claude Eslin, Paris, Editions du Seuil (Points Sagesse), 1994, vol. 3, p. 151.

PL, vol. 41, col. 660 : "Ac per hoc etiam in is rebus, in quibus non apparet divina justitia, salutaris est divina doctrina. Nescimus enim quo judicio Dei bonus ille sit pauper, malus ille sit dives : iste gaudeat, quem pro suis perditis moribus cruciare debuisse mororibus arbitramur ; contristetur ille, quem vita laudabilis gaudere debuisse persuadet". 
voyage le mène au but recherché. Enfin, il intime l'ordre aux marins de laisser le navire dériver au gré des vents. C'est ainsi qu'il arrive sans le savoir dans le pays de sa mère. Contrairement au texte français dont les différentes versions soulignent l'action du diable qui le ramène dans ce pays, Hartmann se garde bien ici d'indiquer qui est à l'œuvre : il ne mentionne ni Dieu, ni le diable, ni même le hasard ou la fortune. Seule la tempête qui conduit Gregorius au pays de sa mère ne présage rien de bon (v. 1839-1841). Ce qui importe avant tout ici, c'est que Gregorius est persuadé qu'il s'en est remis à la volonté de Dieu et qu'il n'a donc qu'à laisser Celui-ci agir, à se laisser porter au gré des flots. Seul le moment de la rencontre entre le jeune homme et la duchesse et des prémisses de l'énamoration rappelle qu'ils sont tous deux victimes "des intrigues de celui qui a séduit Eve lorsqu'elle se détourna des commandements de Dieu” (v. 1960-1963 : "daz macheten sîne ræte / der ouch vroun Êven verriet / dô si von gotes gebote schiet"). Le mariage est également placé sous le signe de l'erreur : tous ceux qui approuvent l'union de Gregorius et de la duchesse croient, à tort, se conformer à la volonté divine et aux préceptes de l'Eglise. Ainsi les barons déclarent-ils que le pays ne peut être défendu par une femme seule contre des agressions injustes. Ils ajoutent que leur duchesse, qui par amour de Dieu a renoncé à se marier, n'agit pas bien et oriente mal sa vie si elle laisse un pays si important dépérir faute d'héritier (Gregorius, v. 2194-2214). Le but recherché par les conseillers est en apparence louable : ils souhaitent que leur suzeraine agisse enfin en fonction des attentes de la société et des commandements divins. Elle doit se conformer aux Ecritures qui, à l'instar de saint Paul (I Cor. 7, 1-9), proclament que le mariage est un état tout à fait honorable pour les laïss :

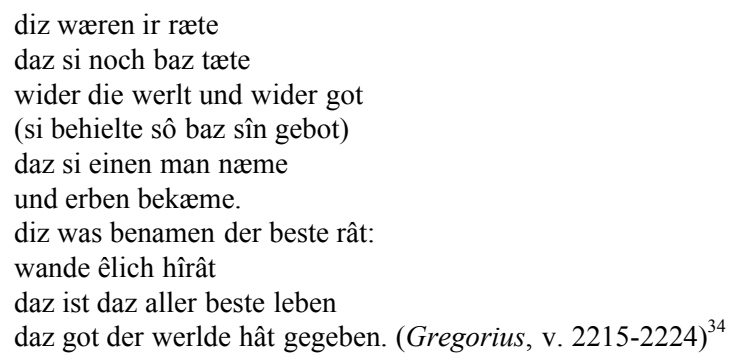

Cette argumentation soignée constitue un ajout de Hartmann. Dans les versions françaises, les barons déclarent simplement qu'ils n'ont jamais vu un homme si jeune d'une telle valeur et qu'il ne pourrait y avoir meilleur seigneur pour un pays ou un comté. Ainsi, ils seraient heureux que leur dame le prenne pour époux $\left(A_{1}\right.$, v. 1547-1554). Ou bien, à la suite des exploits accomplis par Grégoire, ils se contentent de s'écrier : "Dame, cestui pren a

34 “Au contraire, ils lui conseillèrent d'agir bien mieux envers le monde et envers Dieu, de mieux se conformer à ses commandements, et d'épouser un homme afin d'avoir un héritier. C'était vraiment le meilleur conseil que l'on puisse donner, car un mariage conclu en bonne et due forme est la meilleure forme d'existence que Dieu a donnée aux laïcs". 
mari !" ( $B_{1}$, v. 1226, "Dame, prends-le pour mari"), ce à quoi le narrateur s'empresse d'ajouter que c'est le diable, qui veut damner Grégoire, qui les fait parler ainsi.

Chez Hartmann, la dénonciation des pratiques matrimoniales perverties a disparu. Contrairement à la Vie du pape Grégoire, les représentants de la société féodale clouent au pilori toutes les conséquences désastreuses pour la société laïque du célibat de leur suzeraine et l'incitent à se marier. Leur optique est pragmatique et correspond à ce que des vassaux sont en droit d'attendre de leur suzeraine. Cependant, cela ne semble pas correspondre à ce qui, selon Hartmann, aurait pu sauver la duchesse, c'est-à-dire la chasteté, preuve d'un repentir sincère, expression manifeste du désir d'expier sa faute. Il semble bien que, si l'on compare le texte allemand aux versions françaises, il y ait chez Hartmann une réelle revalorisation de l'abstinence sexuelle. Rappelons que dans les versions françaises, la dame, après avoir eu des relations incestueuses avec son frère, refuse de se marier afin de servir Dieu de tout son cœur et de racheter l'âme de son frère. En ce sens, elle s'inflige de nombreux jeûnes, fait des dons aux églises et héberge les pauvres $\left(\mathrm{A}_{1}, \mathrm{v}\right.$. 732$\left.736 ; \mathrm{B}_{1}, \mathrm{v} .568-571\right)$, et n'a donc cure des hommes qui, parce qu'ils convoitent sa terre, viennent lui demander sa main $\left(\mathrm{A}_{1}, \mathrm{v} .724-728, \mathrm{~B}_{1}\right.$, v. 561-564). Hartmann modifie le sens du passage et amplifie l'idée de pénitence. Le choix que la duchesse opère après le premier inceste est assimilé à un mariage avec Dieu et préfigure, en ce sens, son entrée dans les ordres tel qu'il a lieu à la fin de la légende : l'adaptation allemande insiste sur la valeur de l'époux céleste, qualifié d'exemple "de constance" (v. 872, "einen stæten helt") ainsi que de "plus noble de tous les hommes" (v. 873, "den aller tiuristen man"), et sur les intentions sincères qui meuvent la duchesse. Après avoir été victime des ruses du diable, elle n'aspire plus qu'à regagner la grâce de Dieu (v. 890, "sîne hulde") et souhaite exprimer son repentir sincère par des prières, des veilles et des jeûnes. Loin d'être assimilé à une réaction inadéquate et dangereuse pour l'ordre social (les versions françaises évoquent immédiatement l'attaque du duc romain), ce comportement ne peut que plaire à Dieu. Malgré leurs bonnes intentions et leur sagesse, les barons sont donc fondamentalement dans l'erreur. En incitant leur suzeraine à renoncer à l'abstinence sexuelle, ils l'éloignent de Dieu et la jettent dans les bras de son fils et dans les rets du diable ${ }^{35}$. C'est là

35 Cf. Ulrich ERnST, Der 'Gregorius' Hartmanns von Aue. Theologische, p. 151 : "Nachdem sie Gefallen an dem fremden Ritter gefunden hat und ihre Konkupiszenz geweckt ist, folgt sie dem Rat der aquitanischen Magnaten, die ihr aus Staatsräson eine Wiederheirat empfehlen, statt zunächst daran zu denken, daß sie Gott als Buße für den Inzest ein keusches Leben gelobt hat, ja sie ist so mit Blindheit geschlagen, daß sie glaubt, der Bruch des Gelübdes müsse Gott wohlgefällig sein (2225ff.). Verständnishilfen für solche nicht schicksalhafte (wie in dem antiken Ödipusmythos), sondern persönlich verschuldete Blindheit bietet hier wieder die patristische Moraltheologie, für die eine Stellungnahme Gregors des Großen repräsentativ ist, nach welcher der in Sünde gefallene Mensch seine Situation verkennt, die sittliche Qualität seines Handelns falsch beurteilt und, was den Willen Gottes angeht, einem Trugschluß zum Opfer fällt". 
l'une des grandes originalités de Hartmann : il n'hésite pas à mettre en scène des seigneurs courtois et sages, sincèrement mus par de bonnes intentions, qui pourtant provoquent la chute de ceux qu'ils aident et conseillent honnêtement. L'exemple le plus parlant de ce type de conseiller courtois et bien intentionné, nous est donné dans le roman d'Iwein par Gawein : ce parangon des vertus chevaleresques a une conception totalement erronée de la chevalerie et de sa fonction aux yeux de Dieu. En donnant le conseil à son ami Iwein de le suivre, de courir les tournois avec lui afin d'accroître sa gloire, en ajoutant avec sagesse qu'Iwein doit demander à son épouse Laudine de lui accorder un délai raisonnable, il ne fait qu'œuvrer - sans le savoir - à la chute et au malheur de son ami : "Hêr Gâwein sîn geselle / der wart sîn ungevelle" $(\text { Iwein, v. } 3029 \text { s. })^{36}$.

Les barons laissent à leur suzeraine le choix de l'époux, ce qui constitue une autre différence majeure avec le texte français : ils n'avancent pas le nom de Gregorius. Il semble que cette liberté que les barons accordent à leur dame, et qui ne correspondait guère à l'usage féodal, reflète le point de vue de l'Eglise et l'importance que celle-ci accordait à la dilectio, au rôle de l'amour, dans les pratiques matrimoniales. Les défaillances de la société féodale mises en lumière par Anita Guerreau-Jalabert à propos du texte français sont soigneusement évitées par Hartmann. En effet, les barons s'efforcent de faire preuve de sagesse : ils songent au devenir du duché et à la nécessité d'établir un lignage, sans jamais perdre de vue les enseignements de l'Eglise. Ils tentent de réaliser la fusion de la raison d'Etat, de leurs propres intérêts politiques et de la vision de l'Eglise qui, à travers le mariage, veut protéger les laïcs de la luxure. Leurs paroles sont d'ailleurs qualifiées par le narrateur de "juste vérité" (v. 2225, "rehte wârheit"). Ainsi la duchesse se conforme-telle à leur jugement, croyant agir selon la volonté de Dieu :

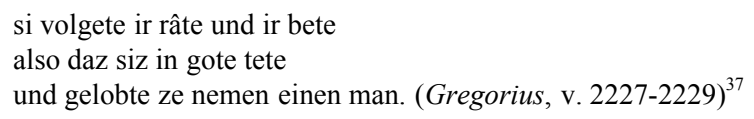

En réfléchissant à l'homme qu'elle pourrait épouser, c'est à Gregorius qu'elle songe, non seulement parce qu'elle est sensible à sa beauté, mais surtout parce qu'elle croit qu'il a été envoyé par Dieu pour la délivrer, elle et son pays (v. 2235-2242). Le narrateur ajoute cependant que c'est ainsi que la volonté du diable fut accomplie (v. 2246). A l'annonce de son intention, tous les barons se réjouissent et reconnaissent Gregorius comme leur seigneur. Celui-ci s'avère d'ailleurs être un seigneur idéal : il se montre très généreux, assure la paix à son pays et en protège les marches de toute agression

36 "Monseigneur Gawein fut la cause de son malheur". Le texte allemand est cité d'après l'édition suivante : HARTMANN VON AUE, Iwein, Text der siebenten Ausgabe von G.F. Benecke, K. Lachmann und L. Wolff. Übersetzung und Anmerkungen von Thomas Cramer, 3. durchgesehene und ergänzte Auflage, Berlin / New York, De Gruyter, 1981.

37 "Elle se conforma à leur conseil et à leur requête et, croyant agir selon Dieu, jura de se marier". 
extérieure. Tous les pays à l'entour auraient pu lui être soumis mais, par crainte de Dieu, il y renonce et s'efforce de toujours faire preuve de juste mesure. Par égard envers Dieu, il ne demande que ce qui lui revient de droit. Gregorius incarne ici l'idéal augustinien du roi juste et pacifique, du souverain soumis à la volonté divine et épris de justice ${ }^{38}$. Ce bonheur apparaît cependant d'emblée comme suspect : alors que chez Hartmann le souverain idéal est secondé traditionnellement par la fortune envoyée par Dieu, la "sælde",39, le terme employé pour désigner le bonheur que vivent Gregorius et son épouse est celui de "wünne"40. Or ce substantif est délibérément ambivalent, car il désigne la joie que procurent à nos sens les plaisirs terrestres. Il a un aspect sensuel qui s'oppose à la signification plus éthérée et positive de "sælde", et rappelle également le bonheur que connaissaient les deux enfants avant le premier inceste (v. 302, "wünne heten si genuoc", "ils furent très heureux"; v. 303, "diese wünne", "ce bonheur"). En effet, cet adjectif apparaît à plusieurs reprises dans la première partie de la légende, alors que le narrateur décrit la beauté parfaite des deux enfants (v. 204 s.) ou lorsque le père, à l'article de la mort, évoque les joies et le bonheur qu'il aurait encore pu connaître avec ses deux enfants s'il lui avait été donné de vivre plus longtemps (v. 217 s.). C'est le même terme qui, dans Le pauvre Henri, désigne les plaisirs mondains auxquels se voue Henri avant d'être puni par Dieu et frappé par la lèpre ${ }^{41}$. Le lecteur ou l'auditeur attentif devine donc que ce bonheur est faux, superficiel, et que l'image du souverain idéal ne sera qu'une parenthèse éphémère, car ce seigneur, malgré ses bonnes intentions, s'est au fond déjà éloigné de Dieu pour les plaisirs futiles du monde qui provoqueront sa "chute brutale" (v. 2262, "einen gæhen val").

Alors que tous - c'est-à-dire les barons, la duchesse et Grégorius - ne songent qu'à agir selon les préceptes divins et optent pour le bien, les subterfuges du diable (v. 2602, "des tiuvels rât") finissent par l'emporter. Ceci explique la colère de Gregorius et les griefs qu'il adresse à Dieu :

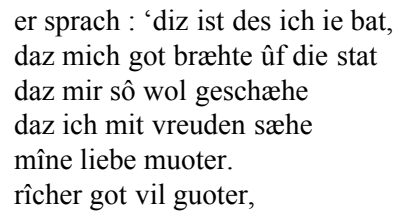

38 Cf. SAInt Augustin, La Cité de Dieu, vol. 1, livre V, chap. XXIV à XXVI, p. 246-250 (De Civitate Dei, PL, vol. 41, col. 170-173).

39 Ceci est particulièrement visible à la fin du roman d'Erec où l'action de la "sælde" est omniprésente.

40 Cf. v. 2251-2253, "ez enwart nie wünne merre / dan diu vrouwe und der herre / mit ein ander hâten" ("jamais il n'y avait eu de plus grande joie que celle que partagèrent la dame et son seigneur") et v. 2260, la vie que mène Gregorius, ce "bon juge" (v. 2257 : "er was guot rihtære"), est qualifiée de "wünnechlîchez leben", de vie pleine de joie.

41 Hartmann Von Aue : Gregorius, Der arme Heinrich, Iwein, herausgegeben und übersetzt von Volker MERTENS, p. 234, v. 79: "werltlîcher wünne" ("plaisirs mondains", on retrouve la même expression également au vers 386 ). 


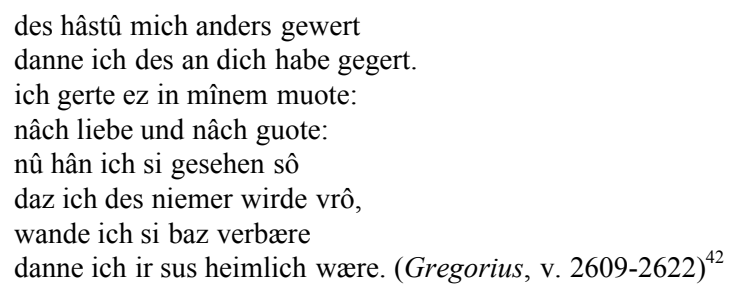

Là encore, Gregorius se trompe et attribue à Dieu la responsabilité du péché qu'il vient de commettre alors que c'était le diable qui était à l'œuvre.

Comme nous l'avons dit plus haut, la perspective de Hartmann est beaucoup plus pessimiste que celle de l'auteur de la Vie du pape Grégoire. Il semble que, dans cette société, le libre arbitre ${ }^{43}$ de l'homme se révèle être un piège : à chaque fois que l'homme croit opter pour le bien et pour Dieu il tombe dans les rets du Malin. L'auteur allemand supprime la critique sociale propre à la légende française pour axer l'action sur des questions existentielles : devant la prédominance du mal, faut-il désespérer de Dieu et de sa justice ? C'est d'ailleurs le thème du désespoir, du péché d'acedia, qui constitue le cœur du prologue de la légende allemande. La réponse est loin d'aller de soi pour le héros qui, comme on le voit, s'emporte contre Dieu. Cette vision pessimiste n'est cependant nullement en contradiction avec l'action de la Providence. Ainsi, lorsque la mère de Gregorius et la femme du baron, qui l'a secondée lors de l'accouchement, ne savent comment sauver le nourrisson, elles s'en remettent à Dieu. Contrairement aux versions françaises, le texte allemand suggère que c'est effectivement Dieu lui-même qui leur inspire d'abandonner le nouveau né aux flots (v. 686-701). C'est encore lui qui protège l'enfant lorsqu'il est seul en mer : Hartmann va ici bien plus loin que la source française et précise que le Christ envoie un vent favorable (v. 787, "den vil rehten wunschwint") à l'enfant qui dérive sur la mer. Il compare Dieu à une nourrice qui prend soin de l'enfant (v. 936, "er was des kindes amme"). Il établit également un parallèle entre Jonas, sauvé par la grâce de Dieu qui pendant trois jours le met à l'abri des vagues dans le ventre d'un "poisson", et l'enfant placé sous la protection divine (v. 929-935). Enfin, Hartmann ajoute que les pleurs de l'enfant, qui permettent à l'abbé de deviner sa présence sur le bateau des pêcheurs, lui sont inspirés par Dieu

\footnotetext{
42 'Il dit: 'C'est ce que $\mathrm{j}$ 'ai toujours demandé : que Dieu me conduise à l'endroit où je pourrais avoir la joie de voir ma chère mère. Dieu, puissant et plein de bonté, tu m'as accordé une chose toute à fait différente de celle que je désirais. En mon for intérieur, je souhaitais des retrouvailles agréables et heureuses. Mais désormais j'ai compris que je ne serai plus jamais heureux, car j'aurais préféré ne jamais la voir plutôt que d'avoir partager avec elle une telle intimité".

43 La question du libre arbitre, du choix pertinent entre le bien et le mal, est soulevée à plusieurs reprises dans le texte : elle apparaît aux vers 1431-1449 (l'abbé explique à Gregorius que Dieu lui a fait don du libre arbitre afin qu'il puisse choisir entre une vie marquée par la honte ou une vie honorable, entre le salut et la damnation) et aux vers 1569-1572 (Gregorius affirme que c'est parce qu'il sait distinguer le bien du mal qu'il choisit la chevalerie, l'erreur s'avérera fatale).
} 
(v. 1014, "von unsers herren minnen"). Comme dans la légende française, c'est encore Dieu qui appelle Gregorius à prendre la place du nouveau pape, qui révèle aux envoyés de Rome qu'ils doivent le chercher dans un endroit désert (v. 3219) et qui opère le miracle permettant au pêcheur de retrouver dans le ventre d'un poisson la clé des chaînes qui entravent le saint homme.

\section{La géographie du péché et de la rédemption}

Cette opposition entre l'action de la Providence et celle du diable s'explique par la géographie des lieux que fréquente Gregorius. Sa première navigation est une navigation bienheureuse qui le mène, alors nourrisson, loin du monde courtois et chevaleresque, loin de la cour d'Aquitaine, et le conduit auprès de l'abbé qui va le recueillir et le baptiser. La seconde navigation, dont le sens n'est pas compris par le jeune Gregorius qui y recherche l'action de la Providence, est celle qui le ramène dans la société courtoise où le jeune homme sera en quête de gloire personnelle et d'honneurs mondains. Enfin, la troisième navigation sera celle qui permettra la fuite du monde et une communion avec Dieu, une union placée sous le signe d'une absolue humilité.

Les lieux et les voyages sont les mêmes que ceux qu'effectue Grégoire dans la légende française mais le sens qu'ils revêtent a changé. Nous ne somme plus dans le schéma mis en lumière par Anita Guerreau-Jalabert (parenté charnelle - parenté spirituelle - union mystique) mais dans un schéma qui oppose une société courtoise, qui croit vivre en adéquation avec les préceptes divins mais qui en réalité est dévoyée, et le monde des hommes de Dieu, c'est-à-dire du moine ou de l'ermite.

Il est tout d'abord surprenant que le registre lexical employé pour désigner les amours incestueux entre le frère et la sœur soit celui qui est propre à l'amour courtois. Hartmann a recours à l'allégorie de dame Amour (substantif féminin en allemand médiéval comme en ancien français) et l'association stéréotypée des plaisirs et de maux d'amour ("lieb" / "leit") :

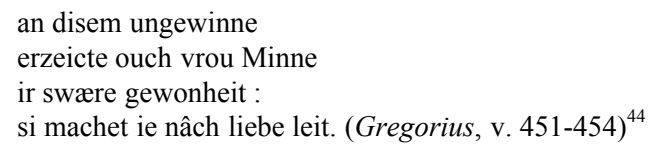

De manière plus étonnante encore, il évoque le topos de l'échange des cœurs - topos que Hartmann a emprunté à Chrétien de Troyes et qu'il tourne en dérision dans Iwein - lorsque le frère prend congé de la sœur :

ein getriuwiu wandelunge ergie,

dô si sich muosen scheiden hie:

44 "Lors de ce malheur, dame Amour montra sa détestable habitude : après les plaisirs survient toujours la souffrance". 
sîn herze volgete ir von dan,

daz ir bestuont bî dem man. (Gregorius, v. 651-654) ${ }^{45}$

La maladie qui emporte le jeune homme est un mal d'amour qui s'empare de lui dès qu'il quitte sa sœur. Le narrateur évoque la puissance des liens de l'amour (v. 834, "des twanc in der minne bant") et la souffrance profonde que lui inflige cet amour (v. 845, "herzeleit"; v. 851, "diu senede nôt" ; v. 852, "herzeriuwe"). A travers le recours à ce registre lexical, inceste et amour courtois semblent mis sur le même plan : les sentiments inspirés par Dame Amour, loin de permettre au chevalier de s'élever moralement comme le prônent troubadours et Minnesänger, s'inscrivent ici dans les plans du diable et se rapportent à une relation pervertie, dévoyée de son sens originel.

L'épisode relatant la vie de Gregorius sur l'île, lieu propice à la fuite du monde et à la communion avec Dieu, constitue une parenthèse heureuse, placée, nous l'avons dit, sous la bienveillante protection de Dieu. Gregorius y trouve un père spirituel (v. 1139, "sîn geistlîch vater") qui le baptise afin qu'il ait "une vie qui plaise à Dieu" (v. 1121, "ein sæliges leben"), le confie à la famille du pêcheur pauvre jusqu'à l'âge de six ans (et non de cinq comme dans les versions françaises) puis l'accueille dans le monastère afin d'en faire un jeune écolier voué aux ordres, un oblat. Dès lors, Gregorius porte l'habit religieux (v. $1161 \mathrm{~s}$.), il reçoit une formation intellectuelle et morale qui lui permet de bientôt rattraper ceux qui ont trois ans de plus que lui. A onze ans, il n'y a pas meilleur grammairien que lui, à quinze ans il maîtrise la théologie avant d'étudier le droit canonique et de devenir un brillant juriste (v. 1196, "ein edel lêgiste"). Cet éloge des capacités intellectuelles de Gregorius peut prêter à confusion. L'auteur suggère-t-il que l'enfant est mû par l'orgueil ? Rien ne le laisse penser : en effet, le jeune homme implore en toute chose la grâce et le conseil de Dieu, et c'est Dieu lui-même qui, ayant doté l'enfant de toutes ces qualités, lui permet d'atteindre la perfection tant intellectuelle que physique (v. 1260-1272). Gregorius est d'ailleurs qualifié d'enfant béni par la fortune (v. 1172, "ein sæligez kint") et consacre tout son esprit à la théologie qui, au Moyen Âge, constitue la science suprême. Même l'attitude de l'abbé qui fait "fructifier" (v. 1105, "[...] erz ze gewinne kêrte") les dix-sept marcs d'or qu'il a gardés (il a donné deux marcs d'or au pêcheur pauvre afin qu'il élève l'enfant et le fasse passer pour son petit-fils, et un marc au pêcheur riche pour qu'il se taise) ne semble pas prêter le flanc à la critique et ne peut être comparée à l'action d'un usurier : il n'a fait que se conformer aux souhaits de la mère de Gregorius (v. 1761-1765) et a sans doute fait fructifier cet or grâce aux richesses que génère le monastère, uniquement pour le bien de Gregorius. Lui aussi apparaît comme un homme béni par la fortune (v. 1102, "der vil sælige man"). La perfection de Gregorius est telle qu'elle laisse présager son origine noble (v. 1273-1284). Ce monde consacré à Dieu et aux études semble être à l'abri du Malin jusqu'au jour où l'enfant blesse en jouant

45 “Un échange sincère eut lieu lorsqu'ils se séparèrent : le cœur du jeune homme la suivit tandis que celui de la jeune femme resta auprès de lui". 
l'un des enfants du pêcheur qui l'a adopté. Hartmann prend soin cependant de se distancer une fois de plus de sa source française et de revaloriser le personnage de Gregorius : il précise qu'il n'a pas agi intentionnellement (v. 1290) et que, profondément attristé par le geste qu'il vient de faire, il suit l'enfant jusqu'à sa maison (v. 1359-1361). Ceci permet également à l'adaptateur de rationaliser sa source : cette modification explique pourquoi Gregorius se trouve alors dans la rue et entend les paroles de la mère qui le traite de "pauvre fou" (v. 1307, "tumber gouch") et de misérable enfant trouvé, amené par le diable (v. 1334-1337).

Cette révélation marque la fin de la période heureuse de Gregorius qui, ne supportant pas de savoir qu'il est un enfant abandonné, décide de quitter le couvent et de se rendre dans un pays où personne n'est au courant de ce qui, à ses yeux, constitue une honte (v. 1426, "jâ vertrîbet mich diu schande", "En vérité, c'est la honte qui me chasse"). L'épisode suivant va être très largement développé par Hartmann qui transforme le bref entretien entre l'abbé et Grégoire en une confrontation entre le représentant du mode de vie monacal, d'une vie contemplative, et le représentant de la chevalerie errante, d'un iuvenis en quête de gloire mondaine et d'honneur ${ }^{46}$. Ce qui dans les différentes versions du texte français ne constitue qu'un court passage opposant la chevalerie à la vie de moine, la mauvaise vie de chevalier à la bonne vie de celui qui se voue aux lettres, à la grammaire et à la religion $\left(\mathrm{A}_{1}\right.$, v. 1112-1136; $B_{1}$, v. 877-898), est considérablement amplifié par Hartmann qui y consacre près de trois cents vers (v. 1432-1738). Ceci montre à quel point cette thématique tient à cœur à l'adaptateur allemand.

A l'abbé qui l'exhorte à ne pas réagir sous l'effet de la colère et à demeurer moine afin, plus tard, de prendre sa place à la tête du monastère, Gregorius répond que son esprit aspire à la chevalerie (v. 1514, "ze ritterschefte stât mîn wân"). Il est prêt à mettre ses facultés au service de la chevalerie : lui qui apprend tout si rapidement saura bien vite développer les compétences nécessaires au métier des armes (v. 1543-1546). Il semble que ce soient les liens du sang, ceux de son haut lignage, qui parlent ici, car sans avoir jamais quitté l'île, sans avoir reçu d'autre éducation qu'un enseignement religieux, Gregorius connaît tout de l'art de la joute : non seulement il rêve de chevalerie mais il maitrise en allemand de nombreux termes techniques propres à cet exercice militaire (v. 1589-1624). Ses paroles restent d'ailleurs totalement incomprises de l'abbé qui les assimile à du grec, langue qui lui est étrangère. Les propos de l'abbé ne constituent pas une condamnation globale de la chevalerie telle que la pratiquait souvent l'Eglise qui assimilait cette caste guerrière au mal (selon l'adage répandu au $\mathrm{XII}^{\mathrm{e}}$ siècle "militia-malitia") mais semble ne viser que les moines qui renoncent à leur état pour se consacrer à la chevalerie. L'abbé affirme que celui qui quitte

46 Cf. Georges DuBY : Les "jeunes" dans la société aristocratique dans la France du nord-ouest au XIII siècle, in : Qu'est-ce que la société féodale?, Paris, Flammarion, 2002, p. $1146-1158$. 
les ordres pour devenir chevalier perdra sa vie et son âme en commentant bien des crimes. Il ajoute que quiconque se détourne de Dieu est voué à l'enfer (v. 1515-1525). Certes, Gregorius n'a pas encore prononcé ses vœux, mais il est malgré tout oblat, il a été recueilli par l'abbé, éduqué dans le monastère et devrait consacrer sa vie à Dieu, son "engagement" étant en principe définitif. A ces arguments, Gregorius répond que la chevalerie est un mode de vie qui peut permettre d'œuvrer à son salut si elle est exercée avec "mesure" (v. 1532, "maze") et qu'il vaut mieux être un "chevalier de Dieu" (v. 1534, "gotes rîter") qu'un moine qui n'a pas la vocation (v. 1535, "ein betrogen klôsterman"). La chevalerie peut donc conduire au salut si elle est bien exercée, si l'on est un chevalier du Christ, c'est-à-dire un guerrier qui met son épée au service du droit et de la protection des faibles. C'est bien ce que sous-entend l'expression "gotes rîter", même si Gregorius ne donne pas davantage de détails sur le sens de cette formule. La définition de la bonne chevalerie donnée ici par Gregorius est juste et pertinente, malheureusement il s'avérera incapable de devenir lui-même un chevalier de Dieu ${ }^{47}$.

Hartmann développe ensuite un autre passage antinomique. L'abbé, qui a fini par céder et par procurer à Gregorius un équipement de chevalier, espère que la crainte de la pauvreté dissuadera son filleul. Au jeune chevalier qui s'apprête à partir à l'aventure, il propose de rester afin de faire un riche mariage. Il pourra alors allier chevalerie et richesse :

nû waz touc dîn riterschaft,

du enhetest guotes die kraft? (Gregorius, v. 1667-1668) ${ }^{48}$

A cette vie de noble casé, s'adonnant au repos (le terme "gemach", désignant le confort qui s'oppose à la quête de l'honneur chevaleresque, est repris aux vers 1670,1680 et 1683), Gregorius déclare préférer la vie d'un chevalier pauvre $^{49}$. Il développe la même idée que celle que Gawein expose à Iwein, dans le roman éponyme, lorsqu'il veut persuader son compagnon, tout juste marié à Laudine, de partir à l'aventure et de courir les tournois en quête de gloire. A l'instar de Gawein, Gregorius déclare qu'une vie dans le confort nuit à la quête de l'honneur : bien des gens fortunés demeurent inactifs tandis que le chevalier pauvre va aspirer à acquérir des biens et sera heureux s'il

47 Cf. W. H. JACKSON, Chivalry in Twelfth-Century. The works of Hartmann von Aue, Arthurian Studies, Cambridge, D. S. Brewer, 1994, p. 152 s.

48 "A quoi te sert-il d'être chevalier si tu es impécunieux ?"

49 Contrairement à ce que suggère W. H. JACKSON (Chivalry in Twelfth-Century. The works of Hartmann von Aue, p. 158 : "Here again Hartmann views knighthood with particular sympathy for the lower end of the hierarchy, in this case the 'arm man' (Gr 1693) who seeks for honourable advancement through the application of effort"), il est sans doute erroné de penser que les paroles du jeune Gregorius reflètent le point de vue de Hartmann tant sa conception de la chevalerie est superficielle et finalement erronée. Voir dans Gregorius un sauveur (ibid., p. 160, "a saviour knight”), un guerrier “qui s'est mis en route dans le plus parfait esprit chevaleresque, c'est-à-dire pour mettre sa chevalerie au service de Dieu" (Brigitte HERLEM-PREY, Le 'Gregorius' et 'La vie de saint Grégoire', p. 81) nous paraît tout aussi contestable et constitue sans doute un contresens sur le texte. 
parvient à accroître sa renommée (v. 1675-1692). De la même façon que Gawein affirme qu'il vaut mieux être un noble pauvre et sans terre qu'un châtelain qui s'adonne à la paresse ${ }^{50}$, Gregorius proclame qu'il n'est pas responsable de sa pauvreté et qu'il porte en lui toutes les terres que son père lui a léguées (v. 1693-1696). Il annonce à l'abbé qu'il ne peut vivre sans honneur et que, puisque ses chevaux sont robustes, ses écuyers preux et courageux, et qu'il est bien équipé, il partira là où il peut acquérir des biens (v. 1714-1729). Cette quête "d'honneur et de biens" (v. 1717, "êre unde guot") prime sur toute autre considération. Gregorius incarne le type du bachelier en quête de gloire, de richesses et d'un bon mariage qui lui permettra de se faire une place dans la société noble et de fonder un lignage. Voyant qu'il ne pourra le dissuader de se consacrer à la chevalerie, l'abbé lui donne les tablettes et lui dévoile ainsi qu'il est effectivement de haute naissance mais que sa conception a été marquée par le péché. Il lui remet également 150 marcs, le produit des dix-sept marcs d'or qu'il a fait fructifier pendant dix-sept ans, souhaitant que le jeune sache lui-même faire accroitre cette somme. Gregorius part donc, avec son équipement de chevalier, ses chevaux et les écuyers qui l'accompagnent ce qui correspond à un groupe tel qu'en formaient effectivement ces chevaliers errants ${ }^{51}$.

Dès lors la grâce divine cesse d'agir, elle n'est plus évoquée par le narrateur, et le diable réaffirme ses droits sur l'âme de Gregorius et celle de sa mère. Lorsqu'il arrive dans le pays de sa mère, c'est bien la quête de gloire et de combat que Gregorius revendique ouvertement. Quand il apprend que la ville, dans laquelle il est arrivé par la mer, est assiégée, il explique aux habitants qui l'ont instruit que c'est exactement ce qu'il a toujours demandé à Dieu. Il souhaitait trouver un endroit dans lequel il ne passerait pas sa jeunesse à être oisif et où règne la guerre. Aussitôt, il propose ses services en tant que mercenaire (v. 1876, "soldenære"). La duchesse le confie à la protection de son sénéchal, qui lui apprend l'art de la guerre et de la joute jusqu'au jour où Gregorius maîtrise parfaitement la technique du combat à la lance et à l'épée (v. 1963-1988). L'amour que lui inspire la beauté de la duchesse l'incite à acquérir "renommée et honneur" (v. 1968, "ûf prîs und ûf êre") : orgueil et inceste semblent indissociables. Ainsi est-il prêt à mettre en jeu ses "maigres biens" et à risquer sa vie pour acquérir la gloire. S'il parvient à vaincre le duc, cette gloire lui sera acquise à jamais (v. 2050-2062).

50 HARTMANN VON AUE, Iwein, v. 2879-2883.

51 L'abbé remplit ici le rôle d'un père qui organise le départ de l'un de ses fils : le chevalier qui partait était généralement accompagné d'un ou deux écuyers et surtout d'un homme de guerre assez expérimenté pour pouvoir protéger le jeune homme non encore aguerri lors des combats. Le rôle de la richesse ne doit pas être surestimé ici (cf. Ulrich ERNST qui insiste sur le riche équipement du chevalier ; Der 'Gregorius' Hartmanns von Aue. Theologische Grundlagen, p. 28) : certes le jeune homme possède une petite fortune en pièces d'or, mais son équipement et le fait que des écuyers l'accompagnent correspondent à la réalité médiévale. Un homme seul n'aurait aucune chance de survie. 
S'il n'incarne pas la chevalerie orgueilleuse et homicide ${ }^{52}$, il n'en demeure pas moins que Gregorius représente une conception totalement erronée de la chevalerie. Il ne met pas son épée au service du droit et de la justice, au service de Dieu, mais ne songe qu'à sa renommée personnelle. Cette quête de vaine gloire est sans cesse soulignée par le texte :

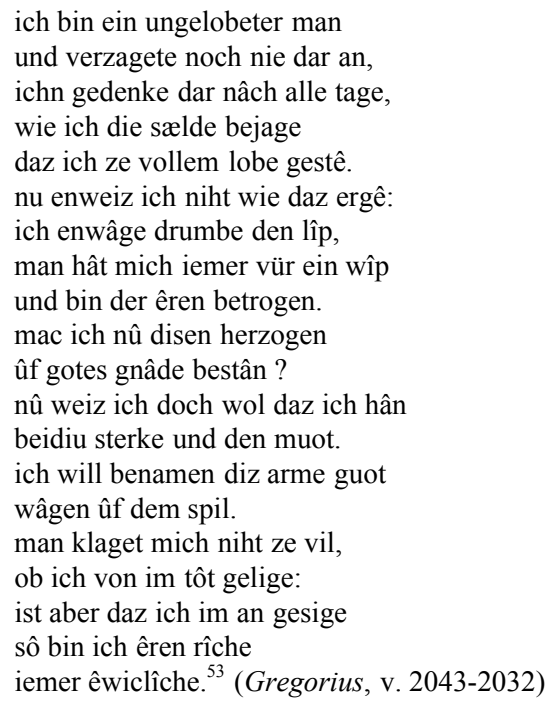

Il n'y a, dès lors, plus rien d'étonnant à ce que le diable puisse refaire son apparition et le prendre dans ses rets. C'est sa quête de gloire qui l'aveugle ${ }^{54}$

52 C'est ce type de chevalier qu'incarne par exemple Mabonagrin dans Erec. Il ne représente pas simplement, comme dans Erec et Enide de Chrétien de Troyes, les dangers d'un amour qui coupe le chevalier de la société mais il renvoie également à la chevalerie homicide : "Je crois que son cœur saignait quand il ne trouvait pas à se battre tant sa main était meurtrière", déclare le narrateur (v. 9021-9023, "ich wæne sîn herze bluote / swenne er nicht ze vehtenne vant: / sô mordic was sîn hant"). Ce chevalier à la taille gigantesque, possédant un cheval et une armure rouges comme le feu, est la personnification de la fureur meurtrière, "représentant des forces diaboliques" (René PÉRENNEC, Recherches sur le roman arthurien en vers en Allemagne aux XII et XIII' siècles, vol. 1: Hartmann von Aue, 'Erec', 'Iwein'. Adaptation et acclimatation, Göppingen, GAG 393 I, 1984 ; ici p. 143.), il constitue sans nul doute une réminiscence du cavalier de l'Apocalypse qui sème la guerre sur la terre entière (Apc. 6,4) en même temps que l'image du cœur qui saigne est une inversion du sacrifice du Christ dont le cœur saigne par charité pour l'humanité.

53 'Je suis un homme dont on ne fait pas l'éloge, pourtant je n'ai de cesse de songer chaque jour au bonheur que cela représenterait pour moi d'être loué de tous. Toutefois, je ne sais comment cela peut être. Si je ne mets pas ma vie en jeu, on me prendra toujours pour une femme et je n'acquerrai jamais d'honneur. Pourrai-je, avec l'aide de la grâce divine, vaincre ce duc ? Je sais bien que j'en ai la force et l'état d'esprit. Par ma foi, je vais mettre en jeu les maigres biens que je possède, et si je meurs personne ne me pleurera. Par contre, si je suis vainqueur, ma gloire sera assurée pour toujours".

54 Le texte souligne la cécité qui marque le cœur de la mère comme du fils, tous deux incapables de se reconnaître : "vür einen gast enpfie si ir kint : / ouch was sîn herze daran blint" (v. 1935 s. : "Elle reçut son fils comme un étranger, tandis que le cœur de celui-ci demeurait 
et le rend insensible aux vertus qui rapprochent de Dieu, à commencer par l'humilité. Ce que Hartmann dénonce ici de manière évidente, c'est l'orgueil qui corrompt fondamentalement la société noble ${ }^{55}$. Le combat qui oppose Gregorius au duc italien s'inscrit d'ailleurs dans cette perspective : chaque chevalier est intimement persuadé d'être le meilleur (v. 1992, "daz er wære der beste" ; v. 2007, "ze dem besten rîter wart genant"). C'était déjà sur l'honneur qui allait lui revenir que le vieux duc mourant avait attiré l'attention de son jeune fils. C'est cette même quête de vanité qui anima ensuite le fils du duc : il offrit à sa sœur tous les habits qu'elle souhaitait et tout ce qui lui faisait plaisir (v. 282-286). Ce qui excite la jalousie du Malin est justement "leur joie et les honneurs dont ils jouissent" (v. 315, "ir vreuden und ir êren"). C'est également au nom de l'honneur que la mère de Gregorius avait subi le viol de son frère : elle avait préféré se soumettre à la volonté du diable plutôt que de perdre son honneur et sa réputation en appelant au secours (v. 385-390). C'est encore pour ne pas mettre leur honneur en péril, que les deux enfants font appel aux conseils d'un homme sage (v. 490-500; v. $528-533)^{56}$. Cette relation entre orgueil et inceste n'a d'ailleurs rien d'étonnant, saint Paul déjà voyait dans l'orgueil la cause de l'inceste et affirmait que ceux qui se livraient à ce péché étaient gonflés d'orgueil ${ }^{57}$. L'orgueil était en effet considéré comme un plus grand péché que la luxure ce qui permettait à certains pères de l'Eglise d'affirmer qu'il était "moins grave de tomber dans l'impureté que de commettre en pensée un péché d'orgueil délibéré, 58 . L'orgueil est la faute qui éloigne de Dieu, qui empêche de contempler la sagesse divine et incite à commettre d'autres péchés.

également aveugle"). Malgré les prières qu'il adresse à Dieu, Gregorius ne saura pas reconnaître la faute dont il se rend coupable jour et nuit avec sa mère (v. 2288-2294). En ce qui concerne ce thème de la caecitas cordis, cf. Ulrich ERNST, Der 'Gregorius' Hartmanns von Aue. Theologische Grundlagen, p. 144-160.

55 Selon Ulrich ERNST, Dieu tolère l'action du diable jusqu'au moment où Gregorius et sa mère, au sommet de leur pouvoir séculier, sont sur le point de succomber au plus important de tous les péchés, l'orgueil (ibid., p. 193 : "So toleriert Gott im Gregorius die Aktionen des Teufels immer dann, wenn die zentralen Figuren - Gregorius und seine Mutter - auf dem Gipfel weltlicher Macht stehen und Gefahr laufen, der Königin der Laster, der superbia, zu verfallen”). Il semble au contraire que Gregorius soit bien aveuglé par l'orgueil lors de cette quête effrénée de gloire et que l'action du diable soit ici évidente, ceci n'empêchera pas toutefois la conversion au bien et à une humilité exemplaire.

56 Cf. Ulrich ERnst, Der 'Gregorius' Hartmanns von Aue. Theologische Grundlagen, p. 5 ss.

57 Cf. I Cor. 5, 1-2 : "omnimo auditur inter vos fornicatio et talis fornicatio qualis nec inter gentes ita ut uxorem patris aliquis habeat et vos inflati esti [...]" ("On n'entend parler que d'inconduite parmi vous, et d'une inconduite telle qu'il n'en existe pas même chez les païens ; c'est à ce point que l'un de vous vit avec la femme de son père! Et vous êtes gonflés d'orgueil !")

58 GrÉGOIRE Le Grand, Moralia in Job $(33,12)$ PL vol. 75, Pars II, col. 688B : “minus est in corporis corruptionem cadere quam cogitatione tacita ex deliberata elatione peccare". Saint Jean Climaque, auteur de L'échelle sainte affirme également qu'il "vaut mieux avoir à combattre la corruption du corps que l'élévation de l'esprit” (PG, vol. 88, col. 709C). 
C'est également un sens aigu de l'honneur qui pousse Gregorius à cacher ses tablettes et à dissimuler ses origines inconnues ainsi que l'inceste dont il est le fruit. Sa réaction, lorsque la duchesse lui demande de lui révéler le secret de sa naissance, nous renseigne sur ses motivations : il croit que quelqu'un l'a accusé d'être de basse extraction (v. 2577, "ein ungeborn man") et affirme qu'il est avec certitude le fils d'un duc. L'importance démesurée qu'il accorde à sa renommée et à la crainte de voir celle-ci amoindrie l'empêche de savoir véritablement qui il est et lui fait perdre de vue la véritable infamie dont il est le résultat. Dans les versions françaises, ce n'est pas la noblesse de Grégoire qui pose problème mais la "grant honte" $\left(\mathrm{A}_{1}\right.$, v. $1809 ; B_{1}$ v. 1456) qui pèse sur lui, la "felonie" $\left(A_{1}\right.$, v. 1834) dont il est le fruit.

Dans une perspective semblable, la femme du pêcheur dont l'enfant a été blessé par le jeune Gregorius, attribue la venue de Gregorius au diable (v. 1134, "der tiuvel hât in her brâht"; "c'est le diable qui l'a amené ici"). Le pêcheur malveillant et cupide, qui emmène Gregorius sur l'île où il vivra en ermite, invoque le diable là où Dieu agira. Ainsi, prenant Gregorius pour un charlatan et non pour un véritable ermite, il le maudit (v. 2808, "daz dir der tiuvel tuo den tôt !"; "que le diable t'emporte !"). Par la suite il affirmera, avant de jeter à la mer la clé des chaînes qui entravent Gregorius, que si ce n'est pas le diable qui, par sa ruse (v. 3091, "mit sînen sinnen"), lui fera quitter ces lieux alors jamais il ne pourra abandonner l'île. Là encore, l'homme est aveuglé par le péché : dans ces deux cas, ce n'est pas l'orgueil qui aveugle le pécheur mais la colère, parfois doublée de l'avarice.

A l'instar de Gawein - qui lui aussi est un représentant des bacheliers, de ces chevaliers errants non casés - Gregorius possède toutes les qualités extérieures du bon chevalier : il est de haute naissance, courageux, probe, noble d'esprit (il ne tue pas son adversaire mais le fait prisonnier), habile au combat (il agit intelligemment afin d'éloigner le duc de l'armée qui pourrait venir à son secours, v. 2102-2111), mais la conception qu'il a de son rôle de chevalier est totalement erronée. Nous sommes bien loin avec lui de l'idéal du miles christianus - comme l'incarnent Erec ou Iwein dans la deuxième partie des romans éponymes - dont l'engagement altruiste et gratuit permet de venir au secours de dames ou de chevaliers dans la détresse. Les termes choisis par Hartmann ont d'ailleurs leur importance : Erec comme Iwein sont toujours secondés par la "sælde", la bonne fortune accordée par Dieu, Gregorius gagne le combat grâce à son art du combat (v. 2138, "kunst") et à la chance ("gelücke"). Le fait qu'il se porte au secours de la duchesse n'est évoqué qu'en dernier lieu : il se battra pour Dieu, pour son honneur et pour délivrer cette femme innocente victime du seigneur lui a dérobé toute sa terre (v. 2070-2074). Il est notable que ces trois éléments soient mis sur le même plan, sans qu'aucune distinction ne soit opérée entre le combat pour Dieu et pour venir en aide à autrui, et la quête de l'honneur personnel. Cette justification n'apparaît qu'après le long passage établissant un parallèle entre le jeu d'échec et le combat chevaleresque (v. 2028-2066) : le combat 
chevaleresque n'est finalement qu'un jeu où l'on peut tout gagner ou tout perdre. Le fait qu'il a délivré sa mère d'un grand malheur est souligné une seconde fois après la défaite du duc romain (v. 2169 s.). Cependant cette action libératrice n'a nullement été le moteur qui a poussé Gregorius à combattre, elle n'en est qu'une conséquence secondaire. Ce qui primait était sans nul doute la quête de la gloire personnelle : sa renommée était déjà grande avant, elle l'est encore davantage maintenant (v. 2171-2174). En tous points, Gregorius représente un groupe social que Hartmann critique également à travers le personnage de Gawein : ces iuvenes qui sont prêts à tout, à perdre leur vie comme le peu de bien qu'ils possèdent, pour acquérir la gloire mondaine et pouvoir épouser un bon parti. La précaution prise jadis par sa mère s'est avérée vaine : en effet, celle-ci avait déposé les tablettes relatant l'inceste dont est issu Gregorius dans le bateau afin qu'il puisse, plus tard, lire les événements qu'elles relatent. Sa mère espérait qu'ainsi il ne serait ne jamais orgueilleux (v. 752, "sô erhüebe er sich niht") et se consacrerait à Dieu et à la prière afin de d'intercéder pour la faute commise par ses parents (v. 746-762). Malgré cette bonne intention, le fait de ne pas inscrire sur les tablettes le pays d'origine de Gregorius ni la lignée à laquelle il appartient (v. 764-766) a fait le jeu du diable.

Les membres de la société courtoise et profane que dépeint ici Hartmann croient pouvoir plaire à Dieu et faire leur salut alors qu'à leur insu ils servent le diable. Ce que Hartmann dénonce ici, c'est une société courtoise dont les valeurs sont fondamentalement faussées, un monde à l'envers dont les membres sont aveuglés par l'orgueil et ne savent distinguer ce qu'ils croient être bien de ce qui véritablement correspond aux attentes de Dieu. Gregorius n'a de cesse d'invoquer Dieu et pourtant il s'en éloigne toujours un peu plus.

Les "artifices du diable" (v. 2602, "des tiuvels râte") semblent donc avoir triomphé de l'âme et du corps des deux époux incestueux. Cependant, la prise de conscience soudaine de la faute commise, qui fait suite à la découverte par la duchesse des tablettes rapportant l'abandon de son fils, provoque chez Gregorius un changement radical d'attitude. Malgré un accès de colère envers Dieu, Gregorius, au lieu de désespérer et de succomber au péché d'acedia ou de tristitia cordis, quitte la cour et la société des hommes pour mener une vie de pénitent et d'ermite. Il se détourne de tout ce qui l'a perdu et rendu aveugle à la volonté de Dieu : il renonce à ses terres, à ses richesses et se défait de son esprit tourné vers le monde (v. 2745-2747; "dem lande und dem guote / und wertlîchem muote / dem sî hiute widerseit"). En ce sens, le texte repose sur une gradation: le séjour de dix-sept ans sur l'île déserte où Gregeorius vit enchaîné à une pierre fait écho aux dix-sept années passées sur l'île où se situe le monastère. La vie d'ermite, le renoncement complet au monde, une communion totale avec Dieu, une survie qui ne dépend plus "que de la bénédiction de Dieu" (v. 3110, "niuwan den gotes segen") et de l'aide accordée par le saint Esprit (v. 3114-3121 ; v. 3469) constituent la phase ultime de la fuite du monde, une existence bien supérieure à la vie contemplative des moines qui, finalement, n'est pas si éloignée des 
considérations du monde. Il suffit, pour noter la différence, de songer à l'argent que l'abbé a fait fructifier ou encore à l'orgueil dont font preuve les moines lorsque le pêcheur leur amène l'enfant afin qu'il soit baptisé (v. 11231126) : sur l'île déserte l'humilité est de mise et les valeurs matérielles ne jouent plus aucun rôle si bien que le pénitent peut, pour reprendre l'expression de saint Jérôme, suivre nu le Christ nu ${ }^{59}$.

En cherchant un lieu désertique où il pourrait se consacrer à Dieu et faire pénitence, il est accueilli par un pêcheur pauvre et méchant. Celui-ci, le prenant pour un charlatan qui veut vivre aux dépends des autres, n'a de cesse de l'humilier. Gregorius endure "le cœur joyeux" (v. 2815 et v. 2946, "mit lachendem muote") les insultes et les vexations que le pêcheur lui inflige et rappelle, par son comportement, l'attitude du fou tel que le dépeint saint Paul :

\footnotetext{
Nous, nous sommes fous à cause du Christ, mais vous vous êtes prudents dans le Christ; nous sommes faibles, mais vous, vous êtes forts ; vous vivez dans l'honneur, mais nous dans le mépris. Jusqu'à l'heure présente, nous avons faim, nous avons soif, nous sommes nus, maltraités et errants; nous nous épuisons à travailler de nos mains. On nous insulte, et nous, nous bénissons ; on nous persécute et nous l'endurons; on nous calomnie, et nous, nous consolons. Nous sommes devenus comme l'ordure du monde, jusqu'à présent l'universel rebut (I Cor. 4, 10-13). ${ }^{60}$
}

Bien plus que les versions françaises, Hartmann met l'accent sur les humiliations, les insultes et les moqueries que lui inflige le pêcheur (v. 2782, "spot" ; v. 2814, "diz schelten" ; v. 2823, "disen spot"; v. 2825, "unwirdikeit"; v. 2826 "swelh vermæcheit unde leit"; v. 2884, "der grozen unwirdikeit" ; v. 2948, "den grôzen spot" ; v. 3644, "hônschaft" ; v. 3668, "hœnlîche"). Le pénitent devient un fou en Dieu, un homme indigne, le contraire de l'homme accompli et noble qu'il était auparavant, et la folie qu'il incarne aux yeux du monde est la vraie sagesse ${ }^{61}$. Ce n'est d'ailleurs pas un hasard si Hartmann insiste sur sa déchéance : lorsque les envoyés de Rome arrivent sur l'île où Gregorius est enchaîné, ils s'attendent à trouver un athlète de Dieu, un "martyr vivant" (v. 3378, "der lebende marterære") dont la beauté serait sans égale, les habits faits d'or et de soie, un homme aux cheveux blonds, au sourire radieux, à la barbe bien coupée comme s'il voulait aller danser (v. 3379-3400). Or ils trouvent un homme velu, à la peau noir, au

59 Eusebius HiERONYMUS : Epistolae (PL vol. 22, Pars V, col. 1085) : "nudum Christum nudus sequere" (Epistola CXXV, Ad rusticum monachum).

60 "Nos stulti propter Christum / vos autem prudentes in Christo / nos infirmi / vos autem fortes / vos nobiles / nos autem ignobiles / usque in hanc horam / et esurimus et sitimus / et nudi sumus / et colaphis caedimur / et instabiles sumus et laboramus operantes manibus nostris / maledicimur et benedicimus / persecutionem patimur et sustinemus / blasphemamur et obsecramus / tanquam purgamenta huius mundi facti sumus".

61 Cf. I Cor. 3, 18-19: "Si quis videtur inter vos sapiens esse in hoc saeculo / stultus fiat ut si sapiens / sapientia enim huius mundi stultitia est apud Deum" ("Si quelqu'un parmi vous croit être sage à la façon de ce monde, qu'il se fasse fou pour devenir sage ; car la sagesse de son monde est folie aux yeux de Dieu"). 
corps émacié, aux orbites creusées, aux yeux rouges, plus rien ne laissant deviner sa beauté passée, ses yeux vivants et clairs, sa bouche rieuse, ses membres musclés et bien faits. Dans cette longue description Hartmann a repris et développé les caractéristiques, présentes dans la source française, qui évoquent un homme sauvage $\left(A_{1}\right.$, v. 2495-2497; $B_{1}$, v. 1941-1944) pour les opposer aux éléments courtois qui jadis faisaient la beauté de Gregorius : tout ce qui chez Gregorius pouvait rappeler le noble, l'homme de cour, beau et enclin aux plaisirs mondains, a disparu. En le présentant durant toute la troisième partie de la légende comme un faible, un homme indigne, sorte de rebut de l'humanité, l'adaptateur allemand fait de Gregorius le paradigme de la grâce divine : il est devenu un homme béni (v. 3241, "den sælderîchen)", l'ami de Dieu (v. 3418, "gotes trût", Hartmann opère d'ailleurs ici une inversion du sens du terme "trût" qui désigne dans la poésie courtoise l'ami charnel, l'amant). L'existence de Gregorius est conforme à celle du Christ crucifié. En martyrisant de la sorte ce corps par lequel il a péché, en s'infligeant des blessures qui ne cicatrisent pas et le font souffrir sans cesse (v. 3449-3458), il fait pénitence et obtient le pardon de Dieu (v. 3418-3422). Le corps est devenu repoussant tandis que l'esprit a été épargné : Gregorius n'a rien perdu de ses facultés intellectuelles ni de son éloquence (v. 34663475). La dichotomie entre le corps répugnant de Gregorius, un corps si abject qu'il est indigne de tout honneur (v. 3551-3552, "der ist sô ungenæme / den êren widerzæme"), et la belle âme du pécheur repenti est bien plus marquée que dans les textes français. C'est justement lorsqu'il se croit rejeté par Dieu et exclu de toute grâce (v. 3539, "der gnâden wære mîn vleisch unwert"; "mon corps est indigne d'une telle grâce") que Gregorius est sauvé et élu par Dieu pour devenir pape. C'est parce qu'il a commis de si terribles fautes, liées à la concupiscence et à l'orgueil, et qu'il les a surmontées que Gregorius va pouvoir, à la tête de la chrétienté, aider les autres fidèles, en commençant par sa propre mère, à expier leurs fautes.

Dans cette troisième partie consacrée à l'existence de pénitent et d'ermite menée par Gregorius, le diable n'a plus aucune place. En effet, les miracles succèdent aux miracles et Hartmann souligne le caractère divin d'événements merveilleux, alors que le rôle de Dieu n'est justement pas explicite dans les versions françaises. C'est, par exemple, Dieu qui indique aux messagers qu'ils trouveront Gregorius dans un lieu sauvage (v. 3219-3222). La découverte des tablettes intactes alors que dix-sept années se sont écoulées et que le pêcheur avait brûlé la cabane qui avait abrité Gregorius (l'inspiration diabolique se manifeste ici clairement à travers l'incendie) est explicitement qualifiée de "très grand signe" (v. 3732, "ein vil grôzes zeichen") envoyé par celui qui est miséricordieux (v. 3730, "der da gnædic ist"). Lors du périple qui les ramène à Rome, Gregorius et ceux qui l'accompagnent sont à l'abri de tout danger tandis que leur réserve de nourriture se reconstitue d'elle-même et que leurs paniers ne sont jamais vides (v. 3741-3752). Il est aisé de constater, à travers tous ses ajouts, que Hartmann ne recule pas devant la surenchère miraculeuse, tout au contraire. Dès lors, l'évocation du diable ne sert plus, en 
fait, qu'à mettre en évidence la grande humilité de Gregorius : ainsi celui-ci déclare-t-il aux deux messagers que c'est le diable qui les envoie afin de le divertir (v. 3575 s.). Enfin, comme dans les versions françaises, les cloches retentissent d'elles-mêmes (v. 3757, “die glocken selben liuten") dès que le groupe de voyageurs approche de la ville sainte et, à peine arrivé, Gregorius guérit de nombreux malades.

Tout aussi miraculeuse est la conversion du pêcheur au bien : après le miracle par lequel la clé des fers de Gregorius est retrouvée dans le ventre d'un poisson, il comprend l'ampleur de la faute qu'il a commise. Toutes les étapes de cette conversion correspondent à celle de la pénitence : il prend conscience de son erreur, s'en repent sincèrement, verse des larmes qui attestent de sa sincérité et qui le lavent de sa faute si bien que son âme est sauvée (v. 3664-3673) ${ }^{62}$. Le pêcheur, qui s'était rendu coupable de tant de péchés mortels échappe donc à l'influence du Malin : un miracle en génère un autre de la même façon que le bien entraîne le bien. La grâce qui touche Gregorius s'étend à tout son entourage. L'auteur se fait le porte-parole du message de l'Eglise et, tel un prédicateur, prône l'importance essentielle que remplit la pénitence si elle est accomplie à temps et si elle est sincère. C'est justement cette question de la bonne pénitence qui est posée par le pêcheur :

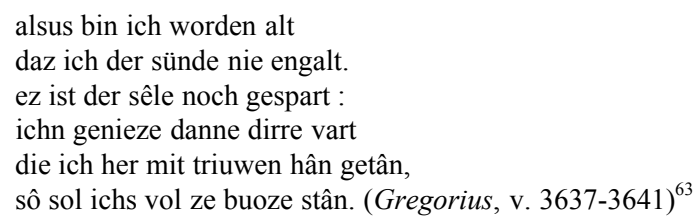

Ce thème du bon repentir et de l'expiation effectuée à temps marquera la prédication de la fin du $\mathrm{XII}^{\mathrm{e}}$ et de tout le XIII ${ }^{\mathrm{e}}$ siècle, tant en France qu'en Allemagne, et elle mènera à l'une des décisions les plus importantes prises lors du IV $\mathrm{IV}^{\mathrm{e}}$ concile de Latran : le décret 21, omnis utriusque sexus, impose la confession et la communion annuelles à tous les laïcs parvenus à "l'âge de discretion" ("annos discretionis"), l'âge lors duquel le chrétien peut distinguer le bien du mal ${ }^{64}$. Cette distinction s'opère soudainement chez le pêcheur qui,

62 A propos de l'importance des larmes comme manifestation du repentir et de leur vertu salutaire, cf. Michel ZINK, La prédication en langue romane avant 1300, Paris, Honoré Champion (Nouvelle bibliothèque du Moyen Âge 4), 1982, p. 445 : "Les larmes sont le signe de la grâce ; elles marquent l'aboutissement de la compassion, la dévotion et la componction. La prière accompagnée de larmes est la plus efficace $[. .$.$] : les larmes de dévotion effacent les$ péchés ; les larmes sont comptées comme mérite et comme vertu ; il faut verser des larmes quand on est en oraison et quand on confesse ses péchés".

63 "Ainsi suis-je devenu vieux sans jamais expier pour ce péché. Et c'est la punition qui attend mon âme : si je ne tire pas les fruits du voyage que j'ai entrepris loyalement ici, alors je devrai expier tout le poids de ma faute".

64 Charles-Joseph HEFELE, Histoire des conciles d'après les documents originaux, tome V, $2^{\text {ème }}$ partie, Paris, A. le Clere, 1913, p. 1349 s. : "Omnis utriusque sexus fidelis, postquam ad annos discretionis pervenerit, omnia sua solus peccata saltem semel in anno fideliter confiteatur proprio sacerdoti, et iniunctam sibi poenitentiam pro viribus studeat adimplere, 
à la vue de la clé, reconnaît "à quel point il était fou" (v. 3307, "wie er getobet hâte").

Ceci rappelle les conseils que donne Gregorius à sa mère avant de la quitter pour mener sa vie d'ermite : il lui demande de ne pas désespérer de Dieu, car le repentir sincère d'un crime a, aux yeux de Dieu, valeur d'expiation. Ainsi sauvera-t-elle son âme dès que ses yeux se rempliront des larmes inspirées par ce repentir. Elle devra renoncer aux mets raffinés et aux vêtements luxueux, fuir le confort et la joie, elle ne devra pas exercer le pouvoir pour l'amour de l'honneur mondain (v. 2713, "durch dehein wertlîch êre") ni pour l'argent mais uniquement pour accomplir la volonté de Dieu. Il lui faudra partager les revenus de ses terres avec les pauvres afin de gagner la miséricorde divine (v. 2728-2730) et fonder des monastères pour apaiser la colère de Dieu. Rappelons que dans les versions françaises, Grégoire demandait juste à sa mère, afin qu'elle obtienne le pardon divin, de jeûner, de prier Dieu, de vivre chastement, de revêtir une haire afin de mortifier son corps, de renoncer aux riches habits, de rendre visite aux moines et de fonder des monastères $\left(\mathrm{A}_{1}\right.$, v. 1973-1994; $\mathrm{B}_{1}$, v. 1585-1606).

Ce thème de la pénitence ouvre d'ailleurs la légende : dans le prologue, le narrateur déclare avoir souvent péché dans sa jeunesse en pensant qu'il pourrait expier ses péchés par la suite. Il associe ses années de jeunesse, qualifiées d'années de folie, de sottise (v. 5, "diu tumben jâren") à l'influence du diable (v. 7, "des helleschergen rât") qui donne aux jeunes gens le conseil suivant :

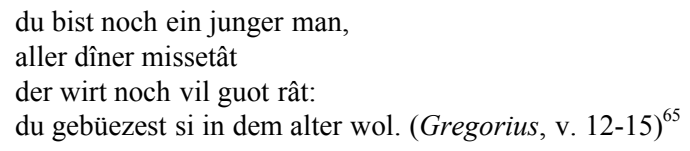

Comme c'est le cas pour le père de Gregorius ainsi que pour Gregorius lui-même, tous deux victimes de leur naïveté juvénile et d'un manque de discernement, la jeunesse s'avère être un allié du diable qui lui permet de parvenir plus facilement à ses fins. Le narrateur indique cependant que bien souvent le pécheur est surpris par la mort qui ne lui laisse pas le temps de faire pénitence. Le même avertissement est fréquemment adressé aux croyants par l'Eglise ${ }^{66}$ et les prédicateurs qui incitent les chrétiens à bien se

suscipiens reverenter ad minus in Pascha Eucharistiae sacramentum" ("Tout fidèle de l'un et l'autre sexe qui a atteint l'âge de raison devra confesser toutes ses fautes au moins une fois chaque année à son propre prêtre, accomplir dans la mesure de ses moyens la pénitence qui lui a été imposée et recevoir dévotement, au moins à Pâques, le sacrement de l'eucharistie").

65 "Tu es encore un jeune homme ; tu pourras encore venir à bout de tous tes méfaits et les expier lorsque tu seras plus âgé".

66 Césaire d'Arles, archevêque du VI ${ }^{\mathrm{e}}$ siècle, posait déjà la question de la valeur de la pénitence accordée au pécheur à l'article de la mort : "Datur quidem etiam in extremis pænitetia, qui non potest denegari : sed auctores tamen nos esse non possumus, quod qui sic petierit mereatur absolvi" (CÉSAIRE D’ARLES, Sermons au peuple, édités et traduits par MarieJosé Delage, Paris, Editions du Cerf, Sources chrétiennes 330, 1986, t. III, p. 84-85 : "En 
préparer à la mort, à ne pas se laisser surprendre et à ne pas se contenter d'un repentir in extremis ${ }^{67}$. Il ajoute qu'aucun péché n'est assez grave pour ne pouvoir être pardonné par Dieu (v. 43-50). L'arme du diable est donc le désespoir : celui-ci s'empare du pécheur qui pense que sa faute est trop lourde $^{68}$. La légende constitue donc une mise en garde, une exhortation destinée à chaque chrétien afin qu'il puisse expier ses fautes de son vivant. L'auteur demande aux croyants de renoncer au doute, au désespoir ${ }^{69}$ qui est à l'origine de la chute de bien des chrétiens (v. 64 s. : "[...] daz er den zwîvel lâze / der manigen versenket"). Il a même recours à la parabole du bon samaritain pour démontrer que Dieu n'abandonne jamais ceux qui conservent l'espoir et la crainte (v. 113, "gedinge unde vorhte"), c'est-à-dire la crainte de la mort et l'espoir d'être sauvé. La faute décrite dans la légende est d'autant plus grave qu'elle est répétée deux fois et se double du péché d'orgueil, mais malgré tout Gregorius comme sa mère parviennent à être sauvés. Le prologue, absent des manuscrits les plus anciens, a sans doute été rajouté plus tard par Hartmann (on le trouve déjà dans l'adaptation latine composée entre 1210 et 1213 par Arnold de Lübeck). Cet ajout correspond fort bien au sens général de la légende et constitue le fil d'Ariane nécessaire à une bonne compréhension du texte.

vérité, on donne, même à toute extrémité, la pénitence, car on ne peut la refuser ; mais pourtant, sans pouvoir garantir que celui qui l'a demandée ainsi mérite d'être absous"). Quant à la valeur d'une telle pénitence, il ne pouvait donner aucune assurance: "Pcenitentiam dare possum, securitatem dare non possum [...]. Dixi non prcesumo, non promitto, nescio plus de Dei voluntate" (Ibid., p. 90-91: "Je peux donner la pénitence, je ne peux donner la sécurité [...]. J'ai dit : je ne présume pas, je ne promets pas, je n'en sais pas plus sur la volonté de Dieu"). Dans le doute, Césaire préconisait de suivre le chemin le plus sûr, c'est-à-dire celui de la pénitence que l'on accomplit lorsque l'on est bien portant.

67 Dans plusieurs exempla, le Stricker, moraliste allemand du début du XIII ${ }^{\mathrm{e}}$ siècle, insiste sur le fait que le chrétien qui a accumulé les péchés sans se repentir, sans se confesser et les expier, se voue délibérément à l'enfer. Il soulève également à plusieurs reprises le problème de la pénitence in extremis et affirme que Dieu ne se laissera pas attendrir par une conversion de dernière minute : une fois devenu vieux, le pécheur impénitent ne renoncera pas sincèrement aux plaisirs du monde mais arrêtera de s'y adonner uniquement parce que la vieillesse et la maladie l'en empêcheront. Le repentir et l'expiation n'ont donc de valeur que s'ils interviennent alors que le pécheur est dans la force de l'âge et en pleine possession de ses moyens physiques et intellectuels. La conversion ne peut s'accomplir sous la contrainte de l'âge mais doit être le résultat d'un choix délibéré du pécheur qui s'engage dorénavant à servir Dieu. L'expiation n'a effectivement de sens que si elle entraîne de vraies souffrances physiques et morales et s'inscrit dans le schéma de l'imitatio Christi. Le martyre du Christ a délivré l'humanité de l'emprise de Satan, car le fils de Dieu est mort au moment de sa vie où il était le plus fort et le plus résistant. Cette idée de la nullité du repentir de dernière minute constitue également une critique de l'hérésie cathare selon laquelle une conversion in extremis permet de passer de l'état de pécheur à celui de "parfait" et rend le salut possible (cf. Die Kleindichtung des Strickers, hrsg. von Wolfgang MOELLEKEN, Göppingen 1973-1978, GAG 107, volumes I à V ; voir en particulier : Processus Luciferi, t. II, $\mathrm{n}^{\circ} 13$; Der altgewordene Sünder, t. III, $\mathrm{n}^{\circ} 104$; Der gefangene Räuber, t. IV, $\mathrm{n}^{\circ} 132$; Die ewige Verdammnis, t. IV, $\mathrm{n}^{\circ} 133$ ).

68 L'exemple biblique pour ce péché de désespoir est fourni par Caïn $(\mathrm{Gn} .4,13)$.

69 Le terme "zwîvel" désigne à la fois le doute et le fait de désespérer (v. 70, "verzwîfeln"), il renvoie à la tristitia cordis ou acedia. 


\section{Conclusion}

Si la puissance du diable paraît plus grande dans le Gregorius que dans $L a$ vie du pape Gregoire, c'est que l'auteur souhaite insister sur la victoire du bien. La tentation diabolique est d'autant plus utile qu'elle semble ne laisser aucune chance à l'homme, qu'elle paraît annihiler tout libre arbitre ou faire de cette faculté une arme du diable, pour finalement permettre au bien de triompher de manière éclatante. Si la défaite du diable n'est pas acquise d'avance comme c'est le cas dans la légende française, la conversion au bien de Gregorius, de sa mère ainsi que du mauvais pêcheur n'en est que plus admirable. Le péché et la tentation diabolique ont permis au pénitent de se purifier : la lutte que le péché entraîne permet la catharsis finale et la victoire sur toute forme de désespoir, de tristitia cordis. L'humilité extrême dont fait preuve Gregorius marque la défaite irréversible du démon: Gregorius a été tenté par le péché d'orgueil, il est tombé dans les rets du diable, la chute l'a si profondément humilié que finalement il est sauvé pour Dieu et perdu pour le démon.

A travers ce texte, ce n'est plus la pratique matrimoniale féodale que l'auteur critique, mais c'est la société courtoise et ses valeurs, son goût du luxe, le rôle que ses membres accordent à l'honneur et à la quête de la gloire ainsi que leur conception erronée de l'amour, d'un amour courtois assimilé à l'inceste. A ces valeurs mondaines, l'auteur oppose l'amour de Dieu et la vie exemplaire de l'ascète, de l'athlète de Dieu. L'aspect religieux et théologique est beaucoup plus développé chez Hartmann que dans les versions françaises : l'homme aveuglé par l'orgueil et la quête de la renommée ne peut, malgré tous ses efforts, que perdre Dieu de vue et opter pour le mal. Dans la sphère mondaine Dieu n'agit plus et seul le diable, usant de ses artifices, semble avoir une influence sur les hommes. Ainsi l'arrière-plan socio-historique n'est-il plus exactement le même que celui de la légende française : Hartmann ne se contente pas de critiquer les valeurs courtoises et profanes en général, il dénonce de manière évidente, à travers le personnage du jeune Gregorius, la vie des bacheliers, des jeunes chevaliers en quête de gloire et d'un bon parti. Pour Hartmann, cette chevalerie est dans l'erreur et semble même, dans le Gregorius, être inspirée par le diable qui profite de cette soif d'honneur et gloire. Tout aussi propre à l'œuvre de Hartmann est également l'importance accordée au rituel de la pénitence dont les différentes phases sont régulièrement évoquées.

De manière surprenante, le message éthique proposé par Hartmann dans Gregorius n'est pas fondamentalement différent de celui dont son roman Iwein, sans doute composé à la même époque, est porteur. Bien qu'il s'agisse de deux textes appartenant à des genres très différents, et même opposés, l'idéologie chrétienne prônée par la légende n'est pas si étrangère à la perspective du roman arthurien. Les points communs ne se limitent pas à la critique de la chevalerie profane et de bacheliers incarnés, nous l'avons dit, par Gregorius ou Gawein. Dans les deux œuvres, Hartmann nous montre, à 
travers Gregorius ou Iwein, l'évolution d'un jeune noble qui renonce à la vaine gloire, à l'orgueil et aux vanitates, pour se vouer à Dieu. Dans le cas de Gregorius, le héros devient un athlète de Dieu, un martyr, un ami de Dieu, un miles Christi qui consacre tous ses efforts à vivre en accord et en communion avec Dieu. Si Gregorius est un soldat spirituel du Christ, Iwein est un miles christianus au sens littéral, un soldat qui met son épée au service des dames et des chevaliers en détresse, luttant contre les forces du mal incarnées par un serpent ou des géants qualifiés de "tiuvels knehte[...]", de valets du diable (Iwein, v. 6338). Il est d'ailleurs notable que tous les deux touchent le fond de la misère humaine en devenant des hommes sauvages, des hommes à l'apparence si répugnante qu'elle s'oppose radicalement à leur vie mondaine passée, à leur beauté ancienne. Si l'ensauvagement d'Iwein est marqué par un véritable accès de folie (le chevalier ne sait plus qui il est et perd toute mémoire des événements passés), Gregorius est quant à lui un fou à cause du Christ. Il s'agit dans les deux cas d'une épreuve cathartique qui permet au héros de se défaire son ancien moi pour renaître à Dieu et aux hommes. L'un devient un chevalier qui, sans cesse aidé et secondé par la grâce divine, met son épée au service du bien et de Dieu, l'autre devient pape et fait régner sur terre la justice divine. Il faut noter que dans Iwein également l'adaptateur allemand se montre critique vis-à-vis de la minne, de l'amour courtois, et supprime de nombreux passages que Chrétiens de Troyes avait consacrés aux vertus de l'amour. Qu'il s'agisse du roman d'/wein ${ }^{70}$ ou des deux légendes composées par Hartmann (Le pauvre Henri et Gregorius), le schéma demeure le même : un jeune noble, ayant une conception erronée de la chevalerie et des devoirs qui lui incombent, se laisse aveugler par la quête de la vaine gloire et oublie Dieu. Les événements l'amèneront cependant à reconnaître son erreur, à s'amender et à apprendre à se comporter toujours avec humilité vis-à-vis de Dieu et des hommes.

70 Le premier roman arthurien adapté par Hartmann, Erec, constitue une exception, car l'idéalisation du héros y est plus poussée : dès le début du roman, Erec fait preuve d'une parfaite humilité et constitue le pendant positif à Iders qui, à l'instar de Mabonagrin, représente la chevalerie orgueilleuse et homicide. 